Monte Carlo Simulation of Macroeconomic Risk with a Continuum Agents: The General Case

Peter J. Hammond and Yeneng Sun

No 803

WARWICK ECONOMIC RESEARCH PAPERS

DEPARTMENT OF ECONOMICS

THE UNIVERSITY OF WARWICK 


\title{
Monte Carlo Simulation of Macroeconomic Risk with a Continuum Agents: The General Case*
}

\author{
Peter J. Hammond ${ }^{\dagger}$ and Yeneng Sun ${ }^{\ddagger}$
}

Revised June 2007

\begin{abstract}
In large random economies with heterogeneous agents, a standard stochastic framework presumes a random macro state, combined with idiosyncratic micro shocks. This can be formally represented by a random process consisting of a continuum of random variables that are conditionally independent given the macro state. However, this process satisfies a standard joint measurability condition only if there is essentially no idiosyncratic risk at all. Based on iteratively complete product measure spaces, we characterize the validity of the standard stochastic framework via Monte Carlo simulation as well as event-wise measurable conditional probabilities. These general characterizations also allow us to strengthen some earlier results related to exchangeability and independence.
\end{abstract}

Keywords: large economy, event-wise measurable conditional probabilities, exchangeability, conditional independence, Monte Carlo convergence, Monte Carlo $\sigma$-algebra, stochastic macro structure.

*Parts of this work were done while Yeneng Sun was visiting Stanford University in July 2003, March-May 2005 and July 2006, and while Peter Hammond was visiting the National University of Singapore in March-April 2004. An early version was presented at the World Congress of the Econometric Society in 2005.

${ }^{\dagger}$ Department of Economics, University of Warwick, Coventry CV4 7AL, U.K. e-mail: peter.hammond@stanford.edu

${ }^{\ddagger}$ Department of Economics, National University of Singapore, 1 Arts Link, Singapore 117570. e-mail: ynsun@nus.edu.sg 


\section{Introduction}

Macroeconomic risks are the common random shocks that influence a significant portion of the population. Reality suggests that these are supplemented by risks at the individual level that influence a negligible portion of the population. Indeed, a standard stochastic framework where many agents face interpersonally correlated risks presumes some kind of random macro state, combined with idiosyncratic micro shocks. ${ }^{1}$ Such a framework can be formally represented by a random process consisting of a continuum of random variables that are conditionally independent given the macro state. As shown in Proposition 3.4 below, however, this process satisfies a standard joint measurability condition only if there is essentially no idiosyncratic risk at all. ${ }^{2}$ So, in the absence of joint measurability, the question is whether one can still find reasonable conditions which guarantee the existence of such a stochastic macro structure.

For the important special case when individual risks are symmetrically distributed, one way of justifying this standard framework is provided in [9] under a special assumption of pairwise measurable probabilities. ${ }^{3}$ Yet often the agents in a macroeconomic model face risks whose probability distributions are affected by non-stochastic individual variables such as location or household type. Then the symmetry assumption is clearly violated. The purpose of this paper is therefore to characterize the validity of the standard stochastic framework for many heterogeneous agents facing individual uncertainty.

As in [9], the approach taken here is inspired by the Monte Carlo method for finding numerical approximations to an ordinary multiple integral by taking the average of the integrand evaluated at randomly selected points. We extend this method in order to simulate macroeconomic uncertainty when many heterogeneous agents face both macroeconomic and individual risks. It is shown that a corresponding Monte Carlo $\sigma$-algebra gives all the non-redundant macro states. Indeed, our Theorem 1 and Proposition 3.2 show that, provided there is weak convergence of the empirical distributions

\footnotetext{
${ }^{1}$ See, for example, [4], [12] and [23] for models with a continuum of agents, or [14], [17], [18] for models with a large finite number of agents.

${ }^{2}$ Proposition 3.4 of this paper generalizes the type of non-measurability result shown for independent random variables in Proposition 1 of [21], and for exchangeable random variables in Proposition 2 of [9]. See [5], [6], [9], [15], [20] and [22] for further discussions of the measurability issue.

${ }^{3}$ See Footnote 9 below or $[9$, p. 750$]$ for a formal definition.
} 
even for just one typical random draw from the agent space, a stochastic macro structure exists.

The rest of the paper is organized as follows. Section 2 of the paper sets out the basic framework. Then Section 3 gives formal statements of our main results. Section 4 focuses on two important special cases. The last Section 5 is an appendix containing all the proofs. They rely on the iteratively complete product measure spaces introduced in the recent paper [11]. In particular, this allows us to remove the special assumption of pairwise measurable probabilities that was used in [9].

\section{Basic formulation}

\subsection{Countably generated sub- $\sigma$-algebras}

Let $(\Omega, \mathcal{A}, P)$ denote a probability space that models all the uncertainty in an economy.

A set $N \subset \Omega$ is null if there exists $A \in \mathcal{A}$ with $P(A)=0$ such that $N \subseteq A$. We assume that $(\Omega, \mathcal{A}, P)$ is complete - i.e., that the $\sigma$-algebra $\mathcal{A}$ includes all null sets. For the rest of the paper, let $\mathcal{C} \subseteq \mathcal{A}$ denote a sub- $\sigma$-algebra of $\mathcal{A}$.

Countably generated sub- $\sigma$-algebras of $\mathcal{A}$, which generalize finitely generated $\sigma$-algebras, will play an important role in this paper because of their ability to represent macroeconomic risk.

Definition 2.1 (1) The relative completion of $\mathcal{C}$ is the smallest $\sigma$-algebra that contains both $\mathcal{C}$ and all the subsets of sets $C \in \mathcal{C}$ such that $P(C)=0$. The completion of $\mathcal{C}$ is the smallest $\sigma$-algebra that contains both $\mathcal{C}$ and all the null sets of $(\Omega, \mathcal{A}, P)$.

(2) The sub- $\sigma$-algebra $\mathcal{C} \subseteq \mathcal{A}$ is countably generated if there is a countable family $\mathcal{F} \subseteq \mathcal{A}$ such that $\mathcal{C}$ is contained in the completion of the $\sigma$-algebra $\sigma(\mathcal{F})$ generated by $\mathcal{F}{ }^{4}$

(3) Given two sub- $\sigma$-algebras $\mathcal{C}, \mathcal{C}^{\prime} \subseteq \mathcal{A}$, say that $\mathcal{C}$ is a sub- $\sigma$-algebra of $\mathcal{C}^{\prime}$, and write $\mathcal{C} \subseteq \mathcal{C}^{\prime}$, if $\mathcal{C}$ is contained in the completion of $\mathcal{C}^{\prime}$.

\footnotetext{
${ }^{4}$ This modifies the terminology of Billingsley [1] by allowing the $\sigma$-algebra generated by a countable set to be completed by the addition of null sets.
} 


\section{$2.2 \quad$ Iteratively complete product spaces}

Let $\left(T_{k}, \mathcal{T}_{k}, \lambda_{k}\right)(k \in \mathbb{N})$ be a sequence of probability spaces. Then

$$
\prod_{k=1}^{n}\left(T_{k}, \mathcal{T}_{k}, \lambda_{k}\right):=\left(\prod_{k=1}^{n} T_{k}, \otimes_{k=1}^{n} \mathcal{T}_{k}, \otimes_{k=1}^{n} \lambda_{k}\right)
$$

is the product of the first $n$ probability spaces, whereas

$$
\prod_{k=1}^{\infty}\left(T_{k}, \mathcal{T}_{k}, \lambda_{k}\right):=\left(\prod_{k=1}^{\infty} T_{k}, \otimes_{k=1}^{\infty} \mathcal{T}_{k}, \otimes_{k=1}^{\infty} \lambda_{k}\right)
$$

is the infinite product of the entire sequence of probability spaces.

In order to state as simply as possible conditions which are necessary as well as sufficient to ensure that macroeconomic risk can be simulated, a stronger form of completion will be used for the products (1) and (2), involving more null sets. The following definition extends what Bledsoe and Morse [2] suggested for the case of two measure spaces — see also Dudley [7, p. 108].

Definition 2.2 The set $E \subseteq \prod_{k=1}^{n} T_{k}$ is said to be iteratively null if for every permutation $\pi$ on $\{1, \ldots, n\}$ one has $\left(t_{1}, t_{2}, \ldots, t_{n}\right) \notin E$ for $\lambda_{\pi(1)}$-a.e. $t_{\pi(1)} \in T_{\pi(1)}, \lambda_{\pi(2)}$-a.e. $t_{\pi(2)} \in T_{\pi(2)}, \ldots, \lambda_{\pi(n)}$-a.e. $t_{\pi(n)} \in T_{\pi(n)}$. That is, for each $k=1, \ldots, n$, the section $E_{t_{k}} \subseteq \prod_{j \neq k} T_{j}$ is iteratively null (or null when $n=2$ ) for $\lambda_{k}$-a.e. $t_{k} \in T_{k}$.

The following two propositions concern product spaces that are completed to include all iteratively null sets. Proofs can be found in [11].

Proposition 2.3 Given any $n \in \mathbb{N}$, let $\mathcal{E}_{n}$ denote the family of all iteratively null sets in $\prod_{k=1}^{n} T_{k}$. Then there exists an iteratively complete product probability space $\left(\prod_{k=1}^{n} T_{k}, \bar{\otimes}_{k=1}^{n} \mathcal{T}_{k}, \bar{\otimes}_{k=1}^{n} \lambda_{k}\right)$ that satisfies the Fubini property, in which:

1. $\bar{\otimes}_{k=1}^{n} \mathcal{T}_{k}$ is the $\sigma$-algebra $\sigma\left(\left[\otimes_{k=1}^{n} \mathcal{T}_{k}\right] \cup \mathcal{E}_{n}\right)$, which is equal to the collection $\left[\otimes_{k=1}^{n} \mathcal{T}_{k}\right] \triangle \mathcal{E}_{n}:=\left\{B \triangle E: B \in \otimes_{k=1}^{n} \mathcal{T}_{k}, E \in \mathcal{E}_{n}\right\}$;

2. $\bar{\otimes}_{k=1}^{n} \lambda_{k}$ is the unique probability measure satisfying $\left[\bar{\otimes}_{k=1}^{n} \lambda_{k}\right](B \triangle E)=$ $\left[\otimes_{k=1}^{n} \lambda_{k}\right](B)$ whenever $B \in \otimes_{k=1}^{n} \mathcal{T}_{k}$ and $E \in \mathcal{E}_{n}$.

Proposition 2.4 There exists an iteratively complete infinite product probability space $\left(\prod_{k=1}^{\infty} T_{k}, \bar{\otimes}_{k=1}^{\infty} \mathcal{T}_{k}, \bar{\otimes}_{k=1}^{\infty} \lambda_{k}\right)$ in which: 
1. $\bar{\otimes}_{k=1}^{\infty} \mathcal{T}_{k}$ is the $\sigma$-algebra generated by the union $\mathcal{G}:=\cup_{n=1}^{\infty} \mathcal{G}_{n}$ of the families $\mathcal{G}_{n}$ of cylinder sets taking the form $A \times \prod_{k=n+1}^{\infty} T_{k}$ for some $n \in \mathbb{N}$ and $A \in \bar{\otimes}_{k=1}^{n} \mathcal{T}_{k}$;

2. $\bar{\otimes}_{k=1}^{\infty} \lambda_{k}$ is the unique countably additive extension to this $\sigma$-algebra of the set function $\mu: \mathcal{G} \rightarrow[0,1]$ defined so that $\mu\left(A \times \prod_{k=n+1}^{\infty} T_{k}\right):=$ $\bar{\otimes}_{k=1}^{n} \lambda_{k}(A)$ for all $A \in \bar{\otimes}_{k=1}^{n} \mathcal{T}_{k}$.

When each probability space $\left(T_{k}, \mathcal{T}_{k}, \lambda_{k}\right)(k \in \mathbb{N})$ is a copy of $(T, \mathcal{T}, \lambda)$, let $\left(T^{n}, \overline{\mathcal{T}}^{n}, \bar{\lambda}^{n}\right)$ and $\left(T^{\infty}, \overline{\mathcal{T}}^{\infty}, \bar{\lambda}^{\infty}\right)$ respectively denote the iterative completions of the $n$-fold and infinite product probability spaces, with typical members denoted by $t^{n}$ and $t^{\infty}$.

\subsection{A Continuum of Random Variables}

Let $X$ be a Polish space (i.e., topologically homeomorphic to a complete separable metric space) with Borel $\sigma$-algebra $\mathcal{B}$. Recall that there must exist a countable $\pi$-system $\mathcal{B}_{\pi}=\left\{B_{m}\right\}_{m=1}^{\infty} \subseteq \mathcal{B}$ (i.e., a family of sets that is closed under finite intersections) that generates the $\sigma$-algebra $\mathcal{B}$.

Let $\mathcal{M}(X, \mathcal{B})$ be the space of Borel probability measures on the Polish space $(X, \mathcal{B})$, equipped with the topology of weak convergence of measures. This makes $\mathcal{M}(X, \mathcal{B})$ itself a Polish space — see, for example, [1, pp. 72-73]. It is noted in Lemma 1 of [9] that a mapping $\mu(\cdot)$ from a probability space to $\mathcal{M}(X, \mathcal{B})$ is measurable if and only if for each $B \in \mathcal{B}$, the real-valued mapping $\mu(\cdot)(B)$ is measurable function on the probability space. These two concepts of measurability are therefore interchangeable.

Let $(T, \mathcal{T}, \lambda)$ be a complete atomless probability space, which we call the index space or space of economic agents. We assume throughout that the economic uncertainty of interest can be modeled as a process $g: T \times \Omega \rightarrow X$ with the property that, for each $t \in T$, the component mapping $\omega \mapsto g_{t}(\omega)$ is measurable, thus making every $g_{t}$ a random variable defined on $(\Omega, \mathcal{A}, P)$ with distribution $P g_{t}^{-1}$ on $(X, \mathcal{B})$ (where $P g_{t}^{-1}(B)=P\left(g_{t}^{-1}(B)\right.$ ) for each $B \in \mathcal{B})$. Let $\mathcal{M}(T \times X, \mathcal{T} \otimes \mathcal{B})$ be the space of probability measures on the measurable space $(T \times X, \mathcal{T} \otimes \mathcal{B})$. 


\section{General Results}

\subsection{Monte Carlo Convergence}

As discussed earlier, the process $g$ is intended to model an economy with many agents who face random shocks at both the macroeconomic and individual level. For this process it is natural to consider the convergence properties of the random variables obtained by taking a random sequential draw from the agent space $T$. Such a general procedure is called "Monte Carlo convergence" in [9].

Given a typical sequential draw $t^{\infty} \in T^{\infty}$, consider the finite sample $t_{1}, t_{2}, \ldots, t_{n}$ for each $n$. The relevant question then is whether, as $n \rightarrow \infty$, there is "Monte Carlo" convergence of the proportion of these $n$ agents for whom either $g(t, \omega)$ or the pair $(t, g(t, \omega))$ belongs to a particular family of sets. Depending on what family of sets we consider, Definition 3.1 below provides several different versions of Monte Carlo convergence. The first involves any set $J$ in the product $\sigma$-algebra $\mathcal{T} \otimes \mathcal{B}$ on $T \times X$. The second only considers product sets $S \times B$ with $S \in \mathcal{T}$ and $B \in \mathcal{B}$. Both concern the pair $(t, g(t, \omega))$. Part (3) of Definition 3.1 is a very special case of parts (1) and (2), whereas part (4) considers convergence in distribution of the random outcomes $g(t, \omega) \in X$.

Definition 3.1 (1) The process $g$ is said to be Monte Carlo convergent if there is a function $\gamma: \Omega \rightarrow \mathcal{M}(T \times X, \mathcal{T} \otimes \mathcal{B})$ such that, for each fixed set $J \in \mathcal{T} \otimes \mathcal{B}$, the mapping $\omega \mapsto \gamma_{\omega}(J)$ is $\mathcal{A}$-measurable, and for $\bar{\lambda}^{\infty}$-a.e. sequence $t^{\infty} \in T^{\infty}$, one has

$$
\frac{1}{n} \sum_{i=1}^{n} 1_{J}\left(t_{i}, g\left(t_{i}, \omega\right)\right) \underset{P-\text { a.s. }}{\longrightarrow} \gamma_{\omega}(J) .
$$

In this case, we say that the mapping $\omega \mapsto \gamma_{\omega}$ is the Monte Carlo limit measure of $g .^{5}$

(2) The process $g$ is said to be Monte Carlo convergent on product sets if there is a Monte Carlo limit function $\gamma: \mathcal{T} \times \mathcal{B} \times \Omega \rightarrow[0,1]$ such that, for each $S \in \mathcal{T}$ and $B \in \mathcal{B}$, and for $\bar{\lambda}^{\infty}$-a.e. sequence $t^{\infty} \in T^{\infty}$, one has

$$
\frac{1}{n} \sum_{i=1}^{n} 1_{S}\left(t_{i}\right) 1_{B}\left(g\left(t_{i}, \omega\right)\right) \underset{P-\text { a.s. }}{\longrightarrow} \gamma(S, B, \omega) .
$$

\footnotetext{
${ }^{5}$ We weaken the corresponding definition in [9] by replacing $\lambda^{\infty}$ with $\bar{\lambda}^{\infty}$.
} 
Then the Monte Carlo $\sigma$-algebra $\mathcal{C}^{g}$ is defined as the smallest $\sigma$-algebra on $\Omega$ w.r.t. which the family of Monte Carlo limit functions $\omega \mapsto \gamma(S, B, \omega)$ $(S \in \mathcal{T}, B \in \mathcal{B})$ are all measurable.

(3) The process $g$ is said to be restricted Monte Carlo convergent if for each $B \in \mathcal{B}$ and for $\bar{\lambda}^{\infty}$-a.e. sequence $t^{\infty} \in T^{\infty}$, the sample average $\frac{1}{n} \sum_{i=1}^{n} 1_{B}\left(g\left(t_{i}, \omega\right)\right)$ converges $P$-a.s. as $n \rightarrow \infty$.

(4) For each $x \in X$, let $\delta_{x}$ denote the degenerate probability measure attaching probability 1 to $x$. Then, given any single randomly drawn sequence $t^{\infty} \in T^{\infty}$ and any $\omega \in \Omega$, for each $n=1,2, \ldots$ the measure defined by $\nu_{t^{\infty}, \omega}^{n}:=\frac{1}{n} \sum_{i=1}^{n} \delta_{g\left(t_{i}, \omega\right)}$ is the empirical distribution on $(X, \mathcal{B})$ generated by the $n$ observations $g\left(t_{i}, \omega\right) \quad(i=1,2, \ldots, n)$. The process $g$ is said to be restricted Monte Carlo convergent in distribution if there exists a random variable $\omega \mapsto \tau_{\omega}$ taking values in the space of Borel probability measures $\mathcal{M}(X, \mathcal{B})$ such that, for $\bar{\lambda}^{\infty}$-a.e. sequence $t^{\infty} \in T^{\infty}$, the empirical distribution $\nu_{t^{\infty}, \omega}^{n}$ converges weakly $P$-a.s. to $\tau_{\omega}$.

The following proposition, showing that all four different versions of Monte Carlo convergence are equivalent, will be proved in Subsection 5.4.

Proposition 3.2 The following conditions are equivalent to each other.

1. The process $g$ is Monte Carlo convergent.

2. The process $g$ is Monte Carlo convergent on product sets.

3. The process $g$ is restricted Monte Carlo convergent.

4. The process $g$ is restricted Monte Carlo convergent in distribution.

\subsection{Stochastic Macro Structure}

Definition 3.3 Let $\mathcal{C}$ be a countably generated sub- $\sigma$-algebra of $\mathcal{A}$.

(1) Two random variables $\phi$ and $\psi$ from $(\Omega, \mathcal{A}, P)$ to $X$ are said to be conditionally independent given $\mathcal{C}$ if, for any Borel sets $B_{1}, B_{2} \in \mathcal{B}$, the conditional probabilities satisfy

$$
P\left(\phi^{-1}\left(B_{1}\right) \cap \psi^{-1}\left(B_{2}\right) \mid \mathcal{C}\right)=P\left(\phi^{-1}\left(B_{1}\right) \mid \mathcal{C}\right) P\left(\psi^{-1}\left(B_{2}\right) \mid \mathcal{C}\right) .
$$

(2) The process $g$ is said to be essentially pairwise conditionally independent given $\mathcal{C}$ if, for $\bar{\lambda}^{2}$-a.e. $\left(t_{1}, t_{2}\right) \in T^{2}$, the random variables $g_{t_{1}}$ and $g_{t_{2}}$ are conditionally independent given $\mathcal{C}$. 
(3) $A \mathcal{T} \otimes \mathcal{C}$-measurable mapping $\mu$ from $T \times \Omega$ to $\mathcal{M}(X, \mathcal{B})$ is said to be an essentially regular conditional distribution process of $g$ if, for $\lambda$ a.e. $t \in T$, the $\mathcal{C}$-measurable mapping $\omega \mapsto \mu_{t \omega}$ is a regular conditional distribution $P\left(g_{t}^{-1} \mid \mathcal{C}\right)$ of the random variable $g_{t}$.

(4) The process $g$ is said to have a stochastic macro structure given $\mathcal{C}$ if $g$ admits an essentially regular conditional distribution process given $\mathcal{C}$, and is essentially pairwise conditionally independent given $\mathcal{C}$. The process $g$ is said to have a stochastic macro structure if there exists a countably generated sub- $\sigma$-algebra $\mathcal{C} \subseteq \mathcal{A}$ such that $g$ has a stochastic macro structure given $\mathcal{C}$. When $\mathcal{C}$ is generated by a random variable $\alpha$ from $(\Omega, \mathcal{A}, P)$ to a Polish space $W$, the elements of $W$ are said to be macro states, and $\alpha$ is said to be a macro state function for the process $g .{ }^{6}$ We say that $\alpha$ is a non-redundant macro state function if $\sigma(\alpha) \subseteq \sigma\left(\alpha^{\prime}\right)$ for any macro state function $\alpha^{\prime}$.

(5) The process $g$ is said to have event-wise measurable conditional probabilities if for each event $A \in \mathcal{A}$ with $P(A)>0$, the function on $T$ that maps $t$ to the conditional probability $P\left(g_{t}^{-1}(B) \mid A\right)$ is $\mathcal{T}$-measurable for each $B \in \mathcal{B}$. This property is obviously equivalent to the requirement that for all $A \in \mathcal{A}$ and $B \in \mathcal{B}$ the mapping $t \mapsto P\left(A \cap g_{t}^{-1}(B)\right)$ is $\mathcal{T}$-measurable.

\subsection{Main Theorem}

The following theorem shows that a process is Monte Carlo convergent if and only if it has a stochastic macro structure, which is also equivalent to it having event-wise measurable conditional probabilities. More specifically, it claims that $\mathcal{C}^{g}$ is countably generated, and essentially the smallest $\sigma$-algebra such that, conditioned on the information represented by $\mathcal{C}^{g}$, the randomness faced by individual economic agents is (essentially) independent. In this sense, the Monte Carlo $\sigma$-algebra $\mathcal{C}^{g}$ represents all the relevant aggregate risk. The proof of the theorem will be given in Subsection 5.4, together with that of Proposition 3.2.

Theorem 1 The following conditions are equivalent to each other.

1. The process $g$ has a stochastic macro structure.

2. The process $g$ is Monte Carlo convergent.

3. The process g has event-wise measurable conditional probabilities.

\footnotetext{
${ }^{6}$ It is well known that a countably generated $\sigma$-algebra is always generated by some random variable; see, for example, Section 2.4 of [9].
} 
Moreover, let $\mathcal{C} \subseteq \mathcal{A}$ be any countably generated $\sigma$-algebra on $\Omega$. Then the process $g$ has a stochastic macro structure given $\mathcal{C}$ if and only if $\mathcal{C}$ contains the Monte Carlo $\sigma$-algebra $\mathcal{C}^{g}$. It follows that any macro state function which generates $\mathcal{C}^{g}$ is non-redundant.

\subsection{Joint measurability implies no idiosyncratic risk}

The following proposition shows that if a standard joint measurability condition is imposed on a process $g$ with a stochastic macro structure, then there is essentially no idiosyncratic risk at all. The proof will be presented in Subsection 5.5 of the Appendix.

Proposition 3.4 If a process $g$ has a stochastic macro structure and is jointly measurable with respect to the usual product $\sigma$-algebra $\mathcal{T} \otimes \mathcal{A}$, then $g_{t}$ is $\mathcal{C}^{g}$-measurable for $\lambda$-almost all $t \in T$.

The following remark illustrates a general way to construct processes that combine nontrivial idiosyncratic with arbitrary macro risk.

Remark 3.5 Let $f$ be any process from $T \times \Omega$ to $\mathbb{R}$ such that the random variables $f_{t}, t \in T$ are essentially pairwise independent. ${ }^{7}$ Let $\mathcal{C}$ be a countably generated sub- $\sigma$-algebra of $\mathcal{A}$. It is well known that it can be generated by a real valued random variable $\theta$ on $(\Omega, \mathcal{A}, P)$; see [1], Ex. 20.1, p. 270. Let $g$ be the process from $T \times \Omega$ to $\mathbb{R}^{2}$ such that $g(t, \omega)=(\theta(\omega), f(t, \omega))$ for each $(t, \omega) \in T \times \Omega$. By Proposition 3 in [11], the random variables $f_{t}(\cdot), t \in T$ are also essentially pairwise conditionally independent given $\mathcal{C}$; so too are the random variables $g_{t}(\cdot), t \in T$, and their transformations $h_{t}\left(g_{t}(\cdot)\right)$, where $h: T \times \mathbb{R}^{2} \rightarrow \mathbb{R}$ is jointly measurable.

\section{Special cases}

The proofs of the results in this section will be presented in Subsection 5.6 of the Appendix.

\subsection{Independent risks}

We first consider the case when $\mathcal{C}^{g}$ is the trivial $\sigma$-algebra $\{\emptyset, \Omega\}$, implying that there is no macro risk.

\footnotetext{
${ }^{7}$ By the classical Kolmogorov Extension Theorem, there exists a collection of independent random variables with a general index set; see, for example, [1] and [6].
} 
The family of random variables $g_{t}(t \in T)$ is said to be essentially pairwise independent if for $\bar{\lambda}^{2}$-a.e. $\left(t_{1}, t_{2}\right) \in T^{2}$ the two random variables $g_{t_{1}}$ and $g_{t_{2}}$ are independent. ${ }^{8}$

In the essential i.i.d. setting, Proposition 1 in [9] relies on the special assumption of pairwise measurable probabilities - i.e., for each $A \in \mathcal{A}$ and $B_{1}, B_{2} \in \mathcal{B}$, the mapping $\left(t_{1}, t_{2}\right) \mapsto P\left(A \cap g_{t_{1}}^{-1}\left(B_{1}\right) \cap g_{t_{2}}^{-1}\left(B_{2}\right)\right)$ is measurable w.r.t. the usual product $\sigma$-algebra $\mathcal{T} \otimes \mathcal{T}$ on the set of pairs $T \times T .{ }^{9}$ The following result generalizes that proposition to the general case of independence without assuming pairwise measurable probabilities.

Proposition 4.1 Let $\mu_{t}$ be a measurable mapping from $(T, \mathcal{T})$ to $\mathcal{M}(X, \mathcal{B})$. The following three conditions are equivalent:

1. the process $g$ is essentially pairwise independent with $\mu_{t}=P g_{t}^{-1}$ for $\lambda$-a.e. $t \in T$;

2. for each $S \in \mathcal{T}$ and $B \in \mathcal{B}$, and for $\bar{\lambda}^{\infty}$-a.e. sequence $t^{\infty} \in T^{\infty}$, one has

$$
\frac{1}{n} \sum_{i=1}^{n} 1_{S}\left(t_{i}\right) 1_{B}\left(g\left(t_{i}, \omega\right)\right) \underset{P-\text { a.s. }}{\longrightarrow} \int_{S} \mu_{t}(B) d \lambda .
$$

That is, $g$ is Monte Carlo convergent on measurable product sets $S \times B$, with Monte Carlo limit function $\gamma(S, B, \omega)=\int_{S} \mu_{t}(B) d \lambda$;

3. the process $g$ is Monte Carlo convergent with Monte Carlo limit measure $\gamma$ on $(T \times X, \mathcal{T} \otimes \mathcal{B})$, defined by $\gamma(J):=\int_{T} \mu_{t}\left(J_{t}\right) d \lambda$ for each $J \in \mathcal{T} \otimes \mathcal{B}$, where $J_{t}$ is t-section of $J$ (i.e. $J_{t}=\{x \in X:(t, x) \in J\}$ ).

One can view the implication $(1) \Longrightarrow(2)$ in Proposition 4.1 as follows. Take any $S \in \mathcal{T}$ and $B \in \mathcal{B}$. Suppose the process $g$ is essentially pairwise independent, with $\mu_{t}=P g_{t}^{-1}$ for $\lambda$-a.e. $t \in T$. Then, for $\bar{\lambda}^{\infty}$-a.e. $t^{\infty} \in T^{\infty}$, the sequence of random variables $g_{t_{i}}(i=1,2, \ldots)$ is mutually independent. ${ }^{10}$ Thus, for $\lambda^{\infty}$-a.e. $t^{\infty} \in T^{\infty}$, the sequence $1_{S}\left(t_{i}\right)\left[1_{B}\left(g_{t_{i}}\right)-\mu_{t_{i}}(B)\right](i=$ $1,2, \ldots)$ of uniformly bounded random variables is mutually independent

\footnotetext{
${ }^{8} \mathrm{~A}$ condition like this is called "almost sure pairwise independence" in [20].

${ }^{9}$ See [9, p. 750]. Note that "pairwise measurable probabilities" is a global condition that was always assumed for the process $g$ in [9], whereas the similar condition of "event-wise measurable conditional probabilities" used here is only one of the equivalent conditions in Theorem 1.

${ }^{10}$ See Proposition 3.4 of [21] and Corollary 1 of [11].
} 
with mean zero; a version of the law of large numbers (see [8], Theorem 8.2 on p. 52) therefore implies that the sequence

$$
\frac{1}{n} \sum_{i=1}^{n} 1_{S}\left(t_{i}\right)\left[1_{B}\left(g_{t_{i}}(\omega)\right)-\mu_{t_{i}}(B)\right] \underset{P-\text { a.s. }}{\longrightarrow} 0
$$

as $n \rightarrow \infty$. On the other hand, the function $1_{B}(t) \mu_{t}(B)$ is $\mathcal{T}$-measurable, so the usual strong law of large numbers implies that for $\lambda^{\infty}$-a.e. $t^{\infty} \in T^{\infty}$, $\frac{1}{n} \sum_{i=1}^{n} 1_{S}\left(t_{i}\right) \mu_{t_{i}}(B)$ converges to $\int_{S} \mu_{t}(B) d \lambda$. Hence, for $\bar{\lambda}^{\infty}$-a.e. sequence $t^{\infty} \in T^{\infty}$,

$$
\frac{1}{n} \sum_{i=1}^{n} 1_{S}\left(t_{i}\right) 1_{B}\left(g\left(t_{i}, \omega\right)\right) \underset{P-\text { a.s. }}{\longrightarrow} \int_{S} \mu_{t}(B) d \lambda .
$$

Thus, the implication $(1) \Longrightarrow(2)$ in Proposition 4.1 is simply an obvious version of the "classical" law of large numbers restated in the continuum setting. What is surprising is that essential pairwise independence is also necessary for this convergence property to hold in this setting - i.e., (2) $\Longrightarrow(1)$, as a converse of the classical law of large numbers. ${ }^{11}$

\subsection{Exchangeable risks}

The second special case arises when the random variables $g_{t}$ are symmetric or exchangeable in the sense that $P\left(A \cap g_{t}^{-1}(B)\right)$ is essentially constant for each $A \in \mathcal{A}$ and $B \in \mathcal{B}$. The following proposition demonstrates that Theorem 1 in [9] is still valid for a general symmetric process $g$, even without the assumption made there of pairwise measurable probabilities.

Proposition 4.2 Suppose $\omega \mapsto \mu_{\omega}$ is a measurable mapping from $(\Omega, \mathcal{A})$ to $\mathcal{M}(X, \mathcal{B})$. Let $\mathcal{C}$ be the $\sigma$-algebra on $\Omega$ which is (countably) generated by this mapping. Then the following conditions are equivalent:

1. for each $A \in \mathcal{A}$ and $B \in \mathcal{B}$, one has $P\left(A \cap g_{t}^{-1}(B)\right)=\int_{A} \mu_{\omega}(B) d P$ for $\lambda$-a.e. $t \in T$;

2. the process $g$ is essentially i.i.d. conditioned on $\mathcal{C}$, with $P\left(g_{t}^{-1} \mid \mathcal{C}\right)=\mu_{\omega}$ for $\lambda$-a.e. $t \in T$;

\footnotetext{
${ }^{11}$ In an extended framework where the process $g$ is jointly measurable with respect to a Fubini extension of the usual product measure-theoretic framework on $T \times \Omega$, Theorem 7.6 of [20], Proposition 3.1 of [21], and Theorem 2.8 in [22] show that essential pairwise independence is necessary as well as sufficient for an exact law of large numbers to hold. Proposition 4.1 here is a counterpart of that result in the sequential or Monte Carlo setting considered in this paper.
} 
3. for each $S \in \mathcal{T}, B \in \mathcal{B}$, and for $\bar{\lambda}^{\infty}$-a.e. sequence $t^{\infty} \in T^{\infty}$, one has

$$
\frac{1}{n} \sum_{i=1}^{n} 1_{S}\left(t_{i}\right) 1_{B}\left(g\left(t_{i}, \omega\right)\right) \underset{P-\text { a.s. }}{\longrightarrow} \lambda(S) \mu_{\omega}(B) ;
$$

4. the process $g$ is Monte Carlo convergent, with Monte Carlo limit given by the product probability measure $\lambda \times \mu_{\omega}$ on $(T \times X, \mathcal{T} \otimes \mathcal{B})$.

For the reader's convenience we recall some basic definitions from [9].

Definition 4.3 (1) The process $g$ is said to be essentially pairwise exchangeable if there exists a common joint probability measure $\pi$ on $(X \times X, \mathcal{B} \otimes \mathcal{B})$ such that almost all pairs of random variables in $\left\{g_{t}: t \in T\right\}$ have the same joint distribution $\pi-i . e$. for $\bar{\lambda}^{2}$-a.e. $\left(t_{1}, t_{2}\right) \in T^{2}$, one has

$$
P\left(g_{t_{1}}^{-1}\left(B_{1}\right) \cap g_{t_{2}}^{-1}\left(B_{2}\right)\right)=\pi\left(B_{1} \times B_{2}\right)=\pi\left(B_{2} \times B_{1}\right)
$$

for all $B_{1}, B_{2} \in \mathcal{B}^{12}$

(2) The process $g$ is said to be essentially symmetric if, for each $A \in \mathcal{A}$ and $B \in \mathcal{B}$, the probability $P\left(A \cap g_{t}^{-1}(B)\right)$ is essentially constant (or in other words, $\lambda$-a.e. independent of $t)$.

(3) Let $\mathcal{C}$ be a countably generated sub- $\sigma$-algebra of $\mathcal{A}$, and let $\mu$ be a $\mathcal{C}$-measurable mapping from $\Omega$ to $\mathcal{M}(X, \mathcal{B})$. The process $g$ is said to be essentially i.i.d. conditioned on $\mathcal{C}$ if $g$ is essentially conditionally independent given $\mathcal{C}$, and for $\lambda$-a.e. $t \in T$, the $\mathcal{C}$-measurable mapping $\omega \mapsto \mu_{\omega}$ is a regular conditional distribution $P\left(g_{t}^{-1} \mid \mathcal{C}\right)$ of the random variable $g_{t}$.

The next proposition generalizes Theorem 2 in [9] by dropping the special assumption of pairwise measurable probabilities.

Proposition 4.4 The following conditions are equivalent:

1. the process $g$ is essentially pairwise exchangeable;

2. the process $g$ is essentially symmetric;

3. there exists a measurable mapping $\omega \mapsto \mu_{\omega}$ from $(\Omega, \mathcal{A})$ to $\mathcal{M}(X, \mathcal{B})$, together with the corresponding countably generated $\sigma$-algebra $\mathcal{C}=$ $\sigma(\mu)$, such that all four equivalent conditions of Proposition 4.2 are satisfied;

4. there exists a countably generated $\sigma$-algebra $\mathcal{C}^{\prime}$ such that the process $g$ is essentially i.i.d. conditioned on $\mathcal{C}^{\prime}$.

\footnotetext{
${ }^{12}$ Since we do not assume pairwise measurable probabilities as in [9], we use the iteratively complete product measure $\bar{\lambda}^{2}$ instead of the usual $\lambda^{2}$.
} 


\section{Appendix: Proofs}

The Appendix is organized as follows. Subsection 5.1 generalizes a result of Talagrand in order to derive an appropriate measurability property from Monte Carlo convergence. Then the properties of a measure-valued mapping $\mu_{t \omega}$ from $T \times \Omega$ to $\mathcal{M}(X, \mathcal{B})$ are systematically studied in Subsection 5.2. The main purpose of that subsection is to obtain a countably generated sub- $\sigma$-algebra of $\mathcal{A}$ that relates to some particular integrals based on $\mu_{t \omega}$. These will be used to identify the stochastic macro structure of $g$ with the Monte Carlo $\sigma$-algebra $\mathcal{C}^{g}$. Preliminary properties of the process $g$ are shown in a series of lemmas in Subsection 5.3. The proofs of Proposition 3.2 and Theorem 1 are presented in Subsection 5.4. The last two subsections present respectively the proof of Proposition 3.4 and of the results in Section 4.

\subsection{A Generalization of Talagrand's result}

The following lemma generalizes Lemma 2.1 in [13, p. 304] to the more general setting of iteratively complete product spaces. We follow the notation in Subsection 2.2.

Lemma 5.1 For each $n \in \mathbb{N}$, let $S_{n}$ be a subset of $T_{n}$ with $\lambda_{n}$-outer measure one. Then the $\bar{\otimes}_{k=1}^{\infty} \lambda_{k}$-outer measure of $\prod_{k=1}^{\infty} S_{k}$ is also one.

Proof: Let $\mathcal{S}_{n}$ denote the $\sigma$-algebra $S_{n} \cap \mathcal{T}_{n}=\left\{S_{n} \cap A \mid A \in \mathcal{T}_{n}\right\}$. Since the outer measure $\lambda_{n}^{*}\left(S_{n}\right)=1$, one can define a countably additive probability measure $\nu_{n}$ on $\left(S_{n}, \mathcal{S}_{n}\right)$ by letting $\nu_{n}\left(S_{n} \cap A\right):=\lambda_{n}(A)$ for all $A \in \mathcal{T}_{n}$. For the sequence of probability spaces $\left(S_{n}, \mathcal{S}_{n}, \nu_{n}\right)$, one can construct the usual product probability spaces and then their iterative completions to obtain $\left(\prod_{k=1}^{n} S_{k}, \bar{\otimes}_{k=1}^{n} \mathcal{S}_{k}, \bar{\otimes}_{k=1}^{n} \nu_{k}\right)$ and $\left(\prod_{k=1}^{\infty} S_{k}, \bar{\otimes}_{k=1}^{\infty} \mathcal{S}_{k}, \bar{\otimes}_{k=1}^{\infty} \nu_{k}\right)$.

For any $n \in \mathbb{N}$, it is clear that the identity

$$
\bar{\otimes}_{k=1}^{n} \nu_{k}\left(F \cap \prod_{k=1}^{n} S_{k}\right)=\bar{\otimes}_{k=1}^{n} \lambda_{k}(F)
$$

holds for any measurable rectangle $F=\prod_{k=1}^{n} A_{k}$ with $A_{k} \in \mathcal{T}_{k}$ for $k=$ $1,2, \ldots, n$. It is obvious that the family $\mathcal{F}_{n}$ of sets $F \in \bar{\otimes}_{k=1}^{n} \mathcal{T}_{k}$ that satisfy Equation (6) is a $\lambda$-class - i.e., it contains $\prod_{k=1}^{n} T_{k}$, as well as all complements and all countable disjoint unions of its members. Since the family of measurable rectangles is a $\pi$-system, the classical Dynkin $\pi-\lambda$ theorem implies that $\otimes_{k=1}^{n} \mathcal{T}_{k} \subseteq \mathcal{F}_{n}-$ see, for example, [1, p. 42]. Now take any iteratively null set $E \in \mathcal{E}_{n}$ as in Proposition 2.3. It is clear that the 
subset $E \cap \prod_{k=1}^{n} S_{k}$ is iteratively null w.r.t. the probability measures $\nu_{k}$ $(k=1, \ldots, n)$. Hence $\mathcal{F}_{n}=\bar{\otimes}_{k=1}^{n} \mathcal{T}_{k}$.

Next, consider the infinite product version of Equation (6) - namely

$$
\bar{\otimes}_{k=1}^{\infty} \nu_{k}\left(F \cap \prod_{k=1}^{\infty} S_{k}\right)=\bar{\otimes}_{k=1}^{\infty} \lambda_{k}(F) .
$$

The previous paragraph shows that Equation (7) holds for every set $F$ in the union $\mathcal{G}=\cup_{n=1}^{\infty} \mathcal{G}_{n}$ of the families $\mathcal{G}_{n}$ of cylinder sets, as defined in Proposition 2.4. By the same Dynkin $\pi-\lambda$ argument as above, Equation (7) holds for any set $F \in \sigma(\mathcal{G})=\bar{\otimes}_{k=1}^{\infty} \mathcal{T}_{k}$. Hence, for any $F \in \bar{\otimes}_{k=1}^{\infty} \mathcal{T}_{k}$ with $\prod_{k=1}^{\infty} S_{k} \subseteq F$, Equation (7) implies that $\bar{\otimes}_{k=1}^{\infty} \lambda_{k}(F)=1$, which shows that the $\bar{\otimes}_{k=1}^{\infty} \lambda_{k}$-outer measure of $\prod_{k=1}^{\infty} S_{k}$ is one.

The following lemma will be used to derive event-wise measurable conditional probabilities from different versions of Monte Carlo convergence in Subsection 5.4. It generalizes to iteratively complete products one part of Theorem 2.4 in [13, p. 310], due to M. Talagrand. Exactly the same proof works here provided that one replaces the use of Lemma 2.1 in [13] by Lemma 5.1 above.

Lemma 5.2 Let $g$ be a real-valued function on $T$. Suppose there is a constant $c$ which, for $\bar{\lambda}^{\infty}$-a.e. sequence $t^{\infty}=\left\{t_{n}\right\}_{n=1}^{\infty} \in T^{\infty}$, satisfies $c=$ $\lim _{n \rightarrow \infty} \frac{1}{n}\left[g\left(t_{1}\right)+\cdots+g\left(t_{n}\right)\right]$. Then $g$ is an integrable function on $(T, \mathcal{T}, \lambda)$.

\subsection{Measure-valued mappings on a product space}

The results in this subsection concern any $\mathcal{T} \otimes \mathcal{A}$-measurable mapping $(t, \omega) \mapsto \mu_{t \omega}$ from $T \times \Omega$ to $\mathcal{M}(X, \mathcal{B})$. Let $\mathcal{B}_{\pi}=\left\{B_{m}\right\}_{m=1}^{\infty} \subseteq \mathcal{B}$ denote a fixed countable $\pi$-system that generates the Borel $\sigma$-algebra $\mathcal{B}$ on $X$.

The following lemma shows that there is a countably generated sub- $\sigma$ algebra $\mathcal{C}^{\prime}$ of $\mathcal{A}$ such that $\mu_{t \omega}$ is $\mathcal{T} \otimes \mathcal{C}^{\prime}$-measurable.

Lemma 5.3 There exists a countable $\pi$-system $\mathcal{C}_{\pi}$ in $\mathcal{A}$ such that, given the completed $\sigma$-algebra $\mathcal{C}^{\prime}$ generated by $\mathcal{C}_{\pi}$, for each set $B \in \mathcal{B}$ the mapping $(t, \omega) \mapsto \mu_{t \omega}(B)$ is $\mathcal{T} \otimes \mathcal{C}^{\prime}$-measurable.

Proof: For each set $B_{m}$ of the countable $\pi$-system $\mathcal{B}_{\pi}$, the mapping $(t, \omega) \mapsto$ $\mu_{t \omega}\left(B_{m}\right)$ is $\mathcal{T} \otimes \mathcal{A}$-measurable. Because $\mathcal{T} \otimes \mathcal{A}$ is generated by the family $\{S \times$ $A \mid S \in \mathcal{T}, A \in \mathcal{A}\}$ of measurable rectangles, it follows that each mapping $(t, \omega) \mapsto \mu_{t \omega}\left(B_{m}\right)$ is measurable w.r.t. the $\sigma$-algebra generated by the family 
$\left\{1_{S \times A} \mid S \in \mathcal{T}, A \in \mathcal{A}\right\}$ of indicator functions. By Theorem 5 in [3, p. 17], each mapping $(t, \omega) \mapsto \mu_{t \omega}\left(B_{m}\right)$ must therefore be measurable w.r.t. the $\sigma$-algebra generated by some countable subfamily $\left\{1_{S_{m k} \times A_{m k}}\right\}_{k=1}^{\infty}$ of these indicator functions, and so by the corresponding countable subfamily $\left\{S_{m k} \times A_{m k}\right\}_{k=1}^{\infty}$ of measurable rectangles. Thus, every mapping $(t, \omega) \mapsto$ $\mu_{t \omega}\left(B_{m}\right)(m=1,2, \ldots)$ must be measurable w.r.t. the common $\sigma$-algebra generated by $\left\{S_{m k} \times A_{m k}\right\}_{m, k=1}^{\infty}$.

Take as $\mathcal{C}_{\pi}$ the countable $\pi$-system $\left\{A_{n}\right\}_{n=1}^{\infty}$ constructed by taking all possible finite intersections of the sets in $\left\{A_{m k}\right\}_{m, k=1}^{\infty}$. Let $\mathcal{C}^{\prime}$ be the completed $\sigma$-algebra generated by $\mathcal{C}_{\pi}$. For each $B_{m} \in \mathcal{B}_{\pi}$, the mapping $(t, \omega) \mapsto$ $\mu_{t \omega}\left(B_{m}\right)$ is $\mathcal{T} \otimes \mathcal{C}^{\prime}$-measurable.

Define $\mathcal{B}^{\prime}$ as the family of Borel sets $B \in \mathcal{B}$ such that the mapping $(t, \omega) \mapsto \mu_{t \omega}(B)$ is $\mathcal{T} \otimes \mathcal{C}^{\prime}$-measurable. This family is a $\lambda$-class that contains the $\pi$-system $\mathcal{B}_{\pi}$. By Dynkin's $\pi-\lambda$ theorem, the family $\mathcal{B}^{\prime}$ is the $\sigma$-algebra generated by $\mathcal{B}_{\pi}$, which is precisely $\mathcal{B}$.

The rest of this subsection obtains a countably generated sub- $\sigma$-algebra $\mathcal{C}^{\mu}$ of $\mathcal{A}$ which is generated by integrals of $\int_{S} \mu_{t \omega} d \lambda$ for suitable measurable sets $S \in \mathcal{T}$. Such a construction will allow us to identify the stochastic macro structure of $g$ with the Monte Carlo $\sigma$-algebra $\mathcal{C}^{g}$ in Subsection 5.4.

Lemma 5.4 Let $\mathcal{T}^{\prime}$ and $\mathcal{A}^{\prime}$ be sub- $\sigma$-algebras of $\mathcal{T}$ and of $\mathcal{A}$ respectively such that, for each set $B \in \mathcal{B}$, the mapping $(t, \omega) \mapsto \mu_{t \omega}(B)$ is $\mathcal{T}^{\prime} \otimes \mathcal{A}^{\prime}$ measurable. Suppose $\mathcal{A}_{\pi}^{\prime}$ is a $\pi$-system that generates $\mathcal{A}^{\prime}$. Let $\mathcal{T}^{\prime \prime}$ be the smallest $\sigma$-algebra on $T$ such that, for every $A^{\prime} \in \mathcal{A}_{\pi}^{\prime}$ and $B \in \mathcal{B}_{\pi}$, the mapping $t \mapsto \int_{A^{\prime}} \mu_{t \omega}(B) d P$ is measurable. Then $\mathcal{T}^{\prime \prime}$ is a sub- $\sigma$-algebra of $\mathcal{T}^{\prime}$, and for each set $B \in \mathcal{B}$, the mapping $(t, \omega) \mapsto \mu_{t \omega}(B)$ is $\mathcal{T}^{\prime \prime} \otimes \mathcal{A}^{\prime}$ measurable.

Proof: First, fix any $A^{\prime} \in \mathcal{A}_{\pi}^{\prime}$ and $B \in \mathcal{B}_{\pi}$. Since the mapping $(t, \omega) \mapsto$ $\mu_{t \omega}(B)$ is $\mathcal{T}^{\prime} \otimes \mathcal{A}^{\prime}$-measurable, the Fubini property implies that the mapping $t \mapsto \int_{A^{\prime}} \mu_{t \omega}(B) d P$ is $\mathcal{T}^{\prime}$-measurable. Hence, $\mathcal{T}^{\prime \prime}$ is a sub- $\sigma$-algebra of $\mathcal{T}^{\prime}$.

Next, we observe that the collection of those $A^{\prime} \in \mathcal{A}^{\prime}$ such that the mapping $t \mapsto \int_{A^{\prime}} \mu_{t \omega}(B) d P$ is $\mathcal{T}^{\prime \prime}$-measurable for every set $B \in \mathcal{B}_{\pi}$ forms a $\lambda$ class that contains the $\pi$-system $\mathcal{A}_{\pi}^{\prime}$. So the collection is $\mathcal{A}^{\prime}$ itself. Therefore, for every $A^{\prime} \in \mathcal{A}^{\prime}$ and $B \in \mathcal{B}_{\pi}$, the mapping $t \mapsto \int_{A^{\prime}} \mu_{t \omega}(B) d P$ is $\mathcal{T}^{\prime \prime}$ measurable.

Now fix any $B \in \mathcal{B}_{\pi}$, and define $h$ as the conditional expectation of $\mu_{t \omega}(B)$ w.r.t. $\mathcal{T}^{\prime \prime} \otimes \mathcal{A}^{\prime}$ - i.e., $h(t, \omega):=\mathbb{E}\left(\mu_{t \omega}(B) \mid \mathcal{T}^{\prime \prime} \otimes \mathcal{A}^{\prime}\right)$. Fix any $A^{\prime} \in$ $\mathcal{A}^{\prime}$. Then the mapping $t \mapsto \int_{A^{\prime}} \mu_{t \omega}(B) d P$ is $\mathcal{T}^{\prime \prime}$-measurable. Because $h$ is 
$\mathcal{T}^{\prime \prime} \otimes \mathcal{A}^{\prime}$-measurable, the Fubini theorem implies that $\int_{A^{\prime}} h(t, \omega) d P$ is $\mathcal{T}^{\prime \prime}$ measurable. Next, let $k(t, \omega):=h(t, \omega)-\mu_{t \omega}(B)$. For any $S^{\prime \prime} \in \mathcal{T}^{\prime \prime}$, the definition of conditional expectation implies that

$$
\int_{S^{\prime \prime}}\left[\int_{A^{\prime}} k(t, \omega) d P\right] d \lambda=\int_{S^{\prime \prime} \times A^{\prime}} k(t, \omega) d \lambda \times P=0 .
$$

Since $\int_{A^{\prime}} k(t, \omega) d P$ is $\mathcal{T}^{\prime \prime}$-measurable, Equation (8) and the arbitrary choice of $S^{\prime \prime} \in \mathcal{T}^{\prime \prime}$ imply that $\int_{A^{\prime}} k(t, \omega) d P=0$ for $\lambda$-a.e. $t \in T$. Therefore, for any $S^{\prime} \in \mathcal{T}^{\prime}$, we have

$$
\int_{S^{\prime} \times A^{\prime}} k(t, \omega) d \lambda \times P=\int_{S^{\prime}}\left[\int_{A^{\prime}} k(t, \omega) d P\right] d \lambda=0 .
$$

Since $\mathcal{T}^{\prime \prime} \subseteq \mathcal{T}^{\prime}$, it is clear that $h$ is $\mathcal{T}^{\prime} \otimes \mathcal{A}^{\prime}$-measurable. So therefore is $k$. Equation (9) and the arbitrary choices of $S^{\prime} \in \mathcal{T}^{\prime}, A^{\prime} \in \mathcal{A}^{\prime}$ then imply that $k(t, \omega)=0$ and so $h(t, \omega)=\mu_{t \omega}(B)$ for $\lambda \times P$-almost all $(t, \omega)$.

Now, the collection $\mathcal{B}^{\prime}$ of those $B \in \mathcal{B}$ for which the mapping $t \mapsto \mu_{t \omega}(B)$ is $\mathcal{T}^{\prime \prime} \otimes \mathcal{A}^{\prime}$-measurable forms a $\lambda$-class that contains the $\pi$-system $\mathcal{B}_{\pi}$. By Dynkin's $\pi-\lambda$ theorem, it follows that $\mathcal{B}^{\prime}=\mathcal{B}$.

By symmetry, we can obtain the following result as in Lemma 5.4.

Lemma 5.5 Let $\mathcal{T}^{\prime}$ and $\mathcal{A}^{\prime}$ be sub- $\sigma$-algebras of $\mathcal{T}$ and $\mathcal{A}$ respectively such that for each set $B \in \mathcal{B}$, the mapping $(t, \omega) \mapsto \mu_{t \omega}(B)$ is $\mathcal{T}^{\prime} \otimes \mathcal{A}^{\prime}$-measurable. Suppose $\mathcal{T}_{\pi}^{\prime}$ is a $\pi$-system that generates $\mathcal{T}^{\prime}$. Let $\mathcal{A}^{\prime \prime}$ be the smallest $\sigma$ algebra on $\Omega$ such that the mapping $\omega \mapsto \int_{S^{\prime}} \mu_{t \omega}(B) d \lambda$ is measurable for every $S^{\prime} \in \mathcal{T}_{\pi}^{\prime}$ and $B \in \mathcal{B}_{\pi}$. Then $\mathcal{A}^{\prime \prime}$ is a sub- $\sigma$-algebra of $\mathcal{A}^{\prime}$, and for each set $B \in \mathcal{B}$ the mapping $(t, \omega) \mapsto \mu_{t \omega}(B)$ is $\mathcal{T}^{\prime} \otimes \mathcal{A}^{\prime \prime}$-measurable.

Definition 5.6 Given the two $\pi$-systems $\mathcal{B}_{\pi}=\left\{B_{m}\right\}_{m=1}^{\infty} \subseteq \mathcal{B}$ and $\mathcal{C}_{\pi}=$ $\left\{A_{n}\right\}_{n=1}^{\infty} \subseteq \mathcal{C}^{\prime}$ as in Lemma 5.3, let $\mathcal{S}^{\mu}:=\sigma\left(\left\{\int_{A_{n}} \mu_{t \omega}\left(B_{m}\right) d P\right\}_{m, n=1}^{\infty}\right)$ be the smallest $\sigma$-algebra on $T$ such that each mapping $t \mapsto \int_{A_{n}} \mu_{t \omega}\left(B_{m}\right) d P$ $(m, n=1,2, \ldots)$ is measurable.

Since $\mathcal{S}^{\mu}$ is countably generated, there is a countable $\pi$-system $\mathcal{S}_{\pi}^{\mu}=$ $\left\{S_{n}\right\}_{n=1}^{\infty} \subseteq \mathcal{S}^{\mu}$ that generates $\mathcal{S}^{\mu}$.

Definition 5.7 Given the two $\pi$-systems $\left\{S_{n}\right\}_{n=1}^{\infty}$ in $\mathcal{T}$ and $\mathcal{B}_{\pi}=\left\{B_{m}\right\}_{m=1}^{\infty}$ in $\mathcal{B}$, let $\mathcal{C}^{\mu}:=\sigma\left(\left\{\int_{S_{n}} \mu_{t \omega}\left(B_{m}\right) d \lambda\right\}_{m, n=1}^{\infty}\right)$ be the smallest $\sigma$-algebra on $\Omega$ such that each mapping $\omega \mapsto \int_{S_{n}} \mu_{t \omega}\left(B_{m}\right) d \lambda(m, n=1,2, \ldots)$ is measurable. 
Lemma 5.8 Fix any $B \in \mathcal{B}$.

(1) The mapping $(t, \omega) \mapsto \mu_{t \omega}(B)$ is $\mathcal{S}^{\mu} \otimes \mathcal{C}^{\mu}$-measurable.

(2) The mapping $\omega \mapsto \int_{S} \mu_{t \omega}(B) d \lambda$ is $\mathcal{C}^{\mu}$-measurable for each $S \in \mathcal{T}$.

Proof: (1) Because of Lemma 5.3, we can apply Lemma 5.4 with $\mathcal{T}^{\prime}=\mathcal{T}$, $\mathcal{A}^{\prime}=\mathcal{C}^{\prime}$ and $\mathcal{A}_{\pi}^{\prime}=\mathcal{C}_{\pi}$; then $\mathcal{T}^{\prime \prime}=\mathcal{S}^{\mu}$. By the Fubini property and the above definition, $\mathcal{S}^{\mu}$ is a sub- $\sigma$-algebra of $\mathcal{T}$. Also, for each set $B \in \mathcal{B}$, the mapping $(t, \omega) \mapsto \mu_{t \omega}(B)$ is $\mathcal{S}^{\mu} \otimes \mathcal{C}^{\prime}$-measurable.

Next, apply Lemma 5.5 with $\mathcal{T}^{\prime}=\mathcal{S}^{\mu}, \mathcal{T}_{\pi}^{\prime}=\mathcal{S}_{\pi}^{\mu}$ and $\mathcal{A}^{\prime}=\mathcal{C}^{\prime}$; then $\mathcal{A}^{\prime \prime}=\mathcal{C}^{\mu}$. The above paragraph and Lemma 5.5 imply that $\mathcal{C}^{\mu}$ is a sub$\sigma$-algebra of $\mathcal{C}^{\prime}$, and for each set $B \in \mathcal{B}$ the mapping $(t, \omega) \mapsto \mu_{t \omega}(B)$ is $\mathcal{S}^{\mu} \otimes \mathcal{C}^{\mu}$-measurable.

(2) Because of (1), the mapping $(t, \omega) \mapsto \mu_{t \omega}(B)$ is $\mathcal{T} \otimes \mathcal{C}^{\mu}$-measurable. So the Fubini property implies that $\omega \mapsto \int_{S} \mu_{t \omega}(B) d \lambda$ is $\mathcal{C}^{\mu}$-measurable for any $S \in \mathcal{T}$.

Lemma 5.9 (1) For P-almost every $\omega \in \Omega$, there is a well-defined probability measure $\gamma_{\omega}^{\mu}$ on $(T \times X, \mathcal{T} \otimes \mathcal{B})$ given by $\gamma_{\omega}^{\mu}(J):=\int_{T} \mu_{t \omega}\left(J_{t}\right) d \lambda$ for all $J \in \mathcal{T} \otimes \mathcal{B}$.

(2) For each $J \in \mathcal{T} \otimes \mathcal{B}$, the mapping $\omega \mapsto \gamma_{\omega}^{\mu}(J)$ is $\mathcal{C}^{\mu}$-measurable.

Proof: (1) As discussed in Subsection 2.3, for each $B \in \mathcal{B}$ the mapping $(t, \omega) \mapsto \mu_{t \omega}(B)$ is $\mathcal{T} \otimes \mathcal{A}$-measurable. The generalized Fubini property in the appendix to [10] implies that $\gamma_{\omega}^{\mu}$ is a well-defined probability measure.

(2) Let $\mathcal{J}$ denote the family of sets $J \in \mathcal{T} \otimes \mathcal{B}$ such that $\omega \mapsto \gamma_{\omega}^{\mu}(J)$ is $\mathcal{C}^{\mu}$-measurable. By (2) in Lemma 5.8, one has $S \times B \in \mathcal{J}$ for any $S \in \mathcal{T}$ and $B \in \mathcal{B}$. But $\mathcal{J}$ is obviously a $\lambda$-class which contains the $\pi$-system $\{S \times B: S \in \mathcal{T}, B \in \mathcal{B}\}$. By Dynkin's $\pi-\lambda$ theorem, $\mathcal{J}$ is the $\sigma$-algebra generated by this $\pi$-system, which implies that $\mathcal{J}=\mathcal{T} \otimes \mathcal{B}$.

\subsection{Preliminary properties of the process}

The following lemma uses the assumption of event-wise measurable conditional probabilities to derive a measure-valued mapping $\mu_{t \omega}$ from $T \times \Omega$ to $\mathcal{M}(X, \mathcal{B})$, to which the results of Subsection 5.2 can therefore be applied.

Lemma 5.10 Assume that for all $A \in \mathcal{A}$ and $B \in \mathcal{B}$, the mapping $t \mapsto$ $P\left(A \cap g_{t}^{-1}(B)\right)$ is $\mathcal{T}$-measurable. Then there is a $\mathcal{T} \otimes \mathcal{A}$-measurable mapping $(t, \omega) \mapsto \mu_{t \omega}$ from $T \times \Omega$ to $\mathcal{M}(X, \mathcal{B})$ such that, for all $A \in \mathcal{A}$ and $B \in \mathcal{B}$,

$$
P\left(A \cap g_{t}^{-1}(B)\right)=\int_{A} \mu_{t \omega}(B) d P \text { for } \lambda \text {-almost all } t \in T .
$$


Moreover any other $\mathcal{T} \otimes \mathcal{A}$-measurable mapping $\mu^{\prime}: T \times \Omega \rightarrow \mathcal{M}(X, \mathcal{B})$ with the same property must satisfy $\mu_{t \omega}^{\prime}=\mu_{t \omega}$ for $(\lambda \times P)$-a.e. $(t, \omega) \in T \times \Omega$.

Proof. By the Borel Isormorphism Theorem, because $X$ is a Polish space, there is a Borel bijection $\phi$ between $X$ and a Borel subset $\phi(X)$ of $\mathbb{R}$ - see [7, Section 13.1], for example. Consider the real-valued process $f=\phi \circ g$. Then it is clear that for all $A \in \mathcal{A}$ and all Borel subsets $B$ of $\mathbb{R}$, the mapping $t \mapsto P\left(A \cap f_{t}^{-1}(B)\right)$ is $\mathcal{T}$-measurable.

Fix any Borel subset $B$ of $\mathbb{R}$. By the usual $\pi-\lambda$ argument, the mapping $t \mapsto P\left(E_{t} \cap f_{t}^{-1}(B)\right)$, which is $\mathcal{T}$-measurable whenever $E$ is a measurable rectangle $S \times A$ with $S \in \mathcal{T}$ and $A \in \mathcal{A}$, is also $\mathcal{T}$-measurable for every $E \in \mathcal{T} \otimes \mathcal{A}$. Evidently the mapping $E \mapsto \nu^{B}(E):=\int_{T} P\left(E_{t} \cap f_{t}^{-1}(B)\right) d \lambda$ is a countably additive measure on $(T \times \Omega, \mathcal{T} \otimes \mathcal{A})$. Evidently $\nu^{B}(E)=0$ whenever $(\lambda \times P)(E)=0$ because then $P\left(E_{t}\right)=0$ for $\lambda$-a.e. $t \in T$. By the Radon-Nikodym theorem, there is a $\mathcal{T} \otimes \mathcal{A}$-measurable function $h^{B}$ from $T \times \Omega$ to $\mathbb{R}_{+}$such that for any $E \in \mathcal{T} \otimes \mathcal{A}$,

$$
\int_{E} h^{B}(t, \omega) d(\lambda \times P)=\int_{T} P\left(E_{t} \cap f_{t}^{-1}(B)\right) d \lambda .
$$

We now follow the standard proof that a regular conditional distribution exists - see, for example, [3, p. 225] or [16, p. 107]. For any $r$ in the set $\mathbb{Q}$ of rational numbers, consider the Borel set $(-\infty, r]$ and the corresponding $\mathcal{T} \otimes \mathcal{A}$-measurable function $h^{(-\infty, r]}$ that satisfies Equation (11). For simplicity, let $\chi_{r}(t, \omega)$ denote the function $h^{(-\infty, r]}(t, \omega)$. After excluding points in a countable collection of $(\lambda \times P)$-null sets, there remains a set $J$ with $(\lambda \times P)(J)=1$ such that, for any $(t, \omega) \in J$ : (i) $r_{1}<r_{2}$ implies that $\chi_{r_{1}}(t, \omega) \leq \chi_{r_{2}}(t, \omega)$; (ii) $\lim _{r \rightarrow q} \chi_{r}(t, \omega)=\chi_{q}(t, \omega)$ for any $q \in \mathbb{Q}$; (iii) $\lim _{r \rightarrow-\infty} \chi_{r}(t, \omega)=0$; (iv) $\lim _{r \rightarrow+\infty} \chi_{r}(t, \omega)=1$.

By the argument of [16, p. 107], for each $(t, \omega) \in J$ there is a probability measure $\tau_{t \omega}$ on $\mathbb{R}$ such that $\tau_{t \omega}((-\infty, r])=\chi_{r}(t, \omega)$ for all $r \in \mathbb{Q}$. Because the function $\chi_{r}$ is $\mathcal{T} \otimes \mathcal{A}$-measurable, so is the mapping $(t, \omega) \mapsto \tau_{t \omega}((-\infty, r])$. This implies that $(t, \omega) \mapsto \tau_{t \omega}(B)$ defines a $\mathcal{T} \otimes \mathcal{A}$-measurable function for each $B \in \mathcal{B}(\mathbb{R})$.

Given any $E \in \mathcal{T} \otimes \mathcal{A}$, Equation (11) implies that

$$
\int_{E} \tau_{t \omega}(B) d(\lambda \times P)=\int_{T} P\left(E_{t} \cap f_{t}^{-1}(B)\right) d \lambda
$$

holds when $B$ is any of the sets $(-\infty, r](r \in \mathbb{Q})$. By the $\pi-\lambda$ Theorem, it follows that Equation (12) also holds for any $E \in \mathcal{T} \otimes \mathcal{A}$ and any Borel set $B \subseteq \mathbb{R}$. 
Because $\phi(X)$ is a Borel set in $\mathbb{R}$, the definition of $f$ implies that

$$
\int_{T} P\left(f_{t}^{-1}(\phi(X))\right) d \lambda=\int_{T} P\left(g_{t}^{-1}(X)\right) d \lambda=1 .
$$

Then applying Equation (12) with $E=T \times \Omega$ and $B=\phi(X)$ gives

$$
\int_{T \times \Omega} \tau_{t \omega}(\phi(X)) d(\lambda \times P)=\int_{T} P\left(f_{t}^{-1}(\phi(X))\right) d \lambda=1,
$$

which implies that $\tau_{t \omega}(\phi(X))=1$ for $(\lambda \times P)$-almost all $(t, \omega) \in T \times \Omega$. Thus there is a $\mathcal{T} \otimes \mathcal{A}$-measurable mapping $\mu$ from $T \times \Omega$ to $\mathcal{M}(X, \mathcal{B})$ such that, for all $B \in \mathcal{B}$, one has $\mu_{t \omega}(B)=\tau_{t \omega}(\phi(B))$ for $(\lambda \times P)$-almost all $(t, \omega) \in T \times \Omega$. Hence

$$
\int_{E} \mu_{t \omega}(B) d(\lambda \times P)=\int_{T} P\left(E_{t} \cap g_{t}^{-1}(B)\right) d \lambda
$$

holds for any $E \in \mathcal{T} \otimes \mathcal{A}$ and any Borel set $B \subseteq \mathbb{R}$.

Fix $A \in \mathcal{A}$ and $B \in \mathcal{B}$. Then for any $S \in \mathcal{T}$, applying Equation (13) with $E=S \times A$ implies that

$$
\int_{S}\left[\int_{A} \mu_{t \omega}(B) d P\right] d \lambda=\int_{S} P\left(A \cap g_{t}^{-1}(B)\right) d \lambda .
$$

But any Radon-Nikodym derivative is essentially unique, so

$$
P\left(A \cap g_{t}^{-1}(B)\right)=\int_{A} \mu_{t \omega}(B) d P \text { for } \lambda \text {-a.e. } t \in T .
$$

Finally, suppose another $\mathcal{T} \otimes \mathcal{A}$-measurable mapping $\mu^{\prime}: T \times \Omega \rightarrow$ $\mathcal{M}(X, \mathcal{B})$ also satisfies Equation (15). Then, for any $S \in \mathcal{T}, A \in \mathcal{A}$ and $B \in \mathcal{B}$, one has

$$
\int_{S} P\left(A \cap g_{t}^{-1}(B)\right) d \lambda=\int_{S \times A} \mu_{t \omega}(B) d(\lambda \times P)=\int_{S \times A} \mu_{t \omega}^{\prime}(B) d(\lambda \times P) .
$$

A familiar application of Dynkin's $\pi-\lambda$ Theorem implies that the equality $\int_{E} \mu_{t \omega}(B) d(\lambda \times P)=\int_{E} \mu_{t \omega}^{\prime}(B) d(\lambda \times P)$ must then hold for every $E \in$ $\mathcal{T} \otimes \mathcal{A}$. The desired result follows from the essential uniqueness of the Radon-Nikodym derivative.

The following lemma, which gives a conditional independence structure for the process $g$, is a special case of Lemma 2 in [11]. 
Lemma 5.11 Let $\mathcal{C} \subseteq \mathcal{A}$ be a countably generated $\sigma$-algebra on $\Omega$. Suppose that $\mu$ is a $\mathcal{T} \otimes \mathcal{C}$-measurable mapping from $(T \times \Omega, \mathcal{T} \otimes \mathcal{A})$ to $\mathcal{M}(X, \mathcal{B})$ which, for each $A \in \mathcal{A}$ and $B \in \mathcal{B}$, satisfies Equation (10). Then the process $g$ is essentially pairwise independent conditioned on $\mathcal{C}$, with $P\left(g_{t}^{-1} \mid \mathcal{C}\right)=\mu_{t \omega}$ for $\lambda$-a.e. $t \in T$.

The next lemma presents an elementary property of the iteratively complete measure $\bar{\lambda}^{\infty}$.

Lemma 5.12 Suppose that $D \subseteq T \times T$ is a $\overline{\mathcal{T}}^{2}$ measurable set satisfying $\bar{\lambda}^{2}(D)=1$. Let $D^{*}$ consist of all sequences $t^{\infty} \in T^{\infty}$ such that $\left(t_{i}, t_{j}\right) \in D$ whenever $(i, j) \in \mathbb{N}$ with $i \neq j$. Then $D^{*} \in \overline{\mathcal{T}}^{\infty}$ and $\bar{\lambda}^{\infty}\left(D^{*}\right)=1$.

Proof: Let $G^{n}$ denote the set of all pairs $(i, j)$ with $i, j \in\{1,2, \ldots, n\}$ satisfying $i \neq j$. For each $n \in \mathbb{N}$ and each pair $(i, j) \in G^{n}$, define $D_{i j}^{n} \subset T^{n}$ as the set of all $t^{n}=\left(t_{i}\right)_{i=1}^{n}$ such that $\left(t_{i}, t_{j}\right) \in D$. Note that $\bar{\lambda}^{n}\left(D_{i j}^{n}\right)=\bar{\lambda}^{2}(D)=1$. Define $D^{n}:=\cap_{(i, j) \in G^{n}} D_{i j}^{n}$, so $T^{n} \backslash D^{n}=\cup_{(i, j) \in G^{n}}\left(T^{n} \backslash D_{i j}^{n}\right)$, and then $\bar{\lambda}^{n}\left(T^{n} \backslash\right.$ $\left.D^{n}\right) \leq \sum_{(i, j) \in G^{n}} \bar{\lambda}^{n}\left(T^{n} \backslash D_{i j}^{n}\right)=0$. It follows that $\bar{\lambda}^{n}\left(D^{n}\right)=1=\bar{\lambda}^{\infty}\left(D^{n} \times\right.$ $\prod_{k=n+1}^{\infty} T_{k}$ ) for each $n \in \mathbb{N}$. But $D^{*}=\cap_{n \in \mathbb{N}}\left[D^{n} \times \prod_{k=n+1}^{\infty} T_{k}\right]$, which implies that $D^{*} \in \overline{\mathcal{T}}^{\infty}$. Moreover $T^{\infty} \backslash D^{*}=\cup_{n \in \mathbb{N}}\left[\left(T^{n} \backslash D^{n}\right) \times \prod_{k=n+1}^{\infty} T_{k}\right]$ and so $\bar{\lambda}^{\infty}\left(T^{\infty} \backslash D^{*}\right) \leq \sum_{n=1}^{\infty} \bar{\lambda}^{n}\left(T^{n} \backslash D^{n}\right)=0$. Hence $\bar{\lambda}^{\infty}\left(D^{*}\right)=1$.

The following lemma, which will be used to derive various versions of Monte Carlo convergence, generalizes Lemma 10 in [9].

Lemma 5.13 Suppose that $g$ is a regular conditionally independent process given $\mathcal{C}$, with $P\left(g_{t}^{-1} \mid \mathcal{C}\right)=\mu_{t \omega}$ for $\lambda$-a.e. $t \in T$. Let $f: T \times X \rightarrow \mathbb{R}$ be any $\mathcal{T} \otimes \mathcal{B}$-measurable function with $\int_{T}\left[\int_{\Omega} f_{t}^{2}\left(g_{t}(\omega)\right) d P\right] d \lambda<\infty$. Then, for $\bar{\lambda}^{\infty}$-a.e. sequence $t^{\infty} \in T^{\infty}$, one has

$$
\frac{1}{n} \sum_{i=1}^{n} f\left(t_{i}, g\left(t_{i}, \omega\right)\right) \underset{P-\text { a.s. }}{\longrightarrow} \int_{T}\left[\int_{X} f(t, x) d \mu_{t \omega}\right] d \lambda=\int_{T \times X} f(t, x) d \gamma_{\omega}^{\mu},
$$

which also implies that $\gamma_{\omega}^{\mu}$ is the Monte Carlo limit $\gamma_{\omega}$ of $g$.

Proof: Given the specified function $f$, we follow the proof of Lemma 10 in [9] and define, for each $t \in T$ and $\omega \in \Omega$, the functions

$$
\psi_{t}(\omega):=f_{t}\left(g_{t}(\omega)\right) ; \varphi(t, \omega):=\int_{X} f_{t}(x) d \mu_{t \omega}(x) ; h_{t}(\omega):=\psi_{t}(\omega)-\varphi_{t}(\omega) .
$$

By hypothesis, $\psi_{t}$ is square-integrable (and so $P$-integrable) on $(\Omega, \mathcal{A}, P)$ for $\lambda$-a.e. $t \in T$. Because $P\left(g_{t}^{-1} \mid \mathcal{C}\right)=\mu_{t \omega}$, the proof of Lemma 10 in [9] shows 
that for $\lambda$-a.e. $t \in T$, one has $\mathbb{E}\left(\psi_{t} \mid \mathcal{C}\right)_{\omega}=\int_{X} f_{t}(x) d \mu_{t \omega}=\varphi(t, \omega)$ for $P$-a.e. $\omega \in \Omega$, and also that $\varphi$ is square integrable w.r.t. $\lambda \times P$.

Because $g$ is assumed to be a regular conditionally independent process, we know that $P\left(\left(g_{t}, g_{t^{\prime}}\right)^{-1} \mid \mathcal{C}\right)(\omega)=\mu_{t \omega} \times \mu_{t^{\prime} \omega}$ for $\bar{\lambda}^{2}$-a.e. $\left(t, t^{\prime}\right) \in T^{2}$. Applying Theorem 1 of $\left[3\right.$, p. 223] to the random variable $\psi_{t} \psi_{t^{\prime}}$, we obtain

$$
\begin{aligned}
\mathbb{E}\left(\psi_{t} \psi_{t^{\prime}} \mid \mathcal{C}\right)_{\omega} & =\int_{X \times X} f_{t}(x) f_{t^{\prime}}(y) d\left(\mu_{t \omega}(x) \times \mu_{t^{\prime} \omega}(y)\right) \\
& =\int_{X} f_{t} d \mu_{t \omega} \int_{X} f_{t^{\prime}} d \mu_{t^{\prime} \omega}
\end{aligned}
$$

for $\bar{\lambda}^{2}$-a.e. $\left(t, t^{\prime}\right) \in T^{2}$, which implies that

$$
\mathbb{E}\left(h_{t} h_{t^{\prime}} \mid \mathcal{C}\right)=\mathbb{E}\left(\psi_{t} \psi_{t^{\prime}} \mid \mathcal{C}\right)-\mathbb{E}\left(\psi_{t} \mid \mathcal{C}\right) \mathbb{E}\left(\psi_{t^{\prime}} \mid \mathcal{C}\right)=0 .
$$

So there exists a $\overline{\mathcal{T}}^{2}$-measurable set $D$ such that $\bar{\lambda}^{2}(D)=1$ and $\mathbb{E}\left(h_{t} h_{t^{\prime}}\right)=0$ for all $\left(t, t^{\prime}\right) \in D$. Now define $D^{*}$ as the set of all sequences $t^{\infty}=\left(t_{i}\right)_{i=1}^{\infty} \in$ $T^{\infty}$ such that $\left(t_{i}, t_{j}\right) \in D$ for all $i, j \in \mathbb{N}$ with $i \neq j$. By Lemma 5.12, $\bar{\lambda}^{\infty}\left(D^{*}\right)=1$. Hence, for all $t^{\infty} \in D^{*}$, the random variables $\left(h_{t_{i}}\right)_{i=1}^{\infty}$ are mutually orthogonal. Arguing as in the last two paragraphs of the proof of Lemma 10 in $[9$, pp. $761-2]$ shows that, for $\bar{\lambda}^{\infty}$-a.e. $t^{\infty} \in T^{\infty}$, one has

$$
\frac{1}{n} \sum_{i=1}^{n} h_{t_{i}}(\omega) \underset{P-\text { a.s. }}{\longrightarrow} 0 \text { and } \frac{1}{n} \sum_{i=1}^{n} \varphi_{t_{i}}(\omega) \underset{P-\text { a.s. }}{\longrightarrow} \int_{T} \varphi_{\omega}(t) d \lambda(t) .
$$

But (17) obviously implies that for $\bar{\lambda}^{\infty}$-a.e. $t^{\infty} \in T^{\infty}$,

$$
\frac{1}{n} \sum_{i=1}^{n} \psi_{t_{i}}(\omega)=\frac{1}{n} \sum_{i=1}^{n} h_{t_{i}}(\omega)+\frac{1}{n} \sum_{i=1}^{n} \varphi_{t_{i}}(\omega) \underset{P-\text { a.s. }}{\longrightarrow} \int_{T} \varphi_{\omega}(t) d \lambda(t) .
$$

Because $\int_{T} \varphi_{\omega}(t) d \lambda(t)=\int_{T}\left[\int_{X} f_{t}(x) d \mu_{t \omega}(x)\right] d \lambda=\int_{T \times X} f(t, x) d \gamma_{\omega}^{\mu}$, the result follows from (18).

\subsection{Proof of Proposition 3.2 and Theorem 1}

We shall prove the equivalence of all the conditions in both Proposition 3.2 and Theorem 1 together. Let $\mathrm{P}(1), \mathrm{P}(2), \mathrm{P}(3)$ and $\mathrm{P}(4)$ indicate parts (1), (2), (3) and (4) of Proposition 3.2, respectively. Similarly, let T(1), T(2) and $\mathrm{T}(3)$ indicate the respective parts of Theorem 1 . Note that $\mathrm{P}(1)$ and $\mathrm{T}(2)$ are identical.

$\mathrm{P}(1) \Longrightarrow \mathrm{P}(2)$ : This is trivial. 
$\mathrm{P}(2) \Longrightarrow \mathrm{P}(3)$ : This is also trivial.

$\mathrm{P}(3) \Longrightarrow \mathrm{T}(3)$ : For each $B \in \mathcal{B}$, let $\varphi^{B}$ be the random variable defined so that for $\bar{\lambda}^{\infty}$-a.e. $t^{\infty} \in T^{\infty}$, one has

$$
\frac{1}{n} \sum_{i=1}^{n} 1_{B}\left(g\left(t_{i}, \omega\right)\right) \underset{P-\text { a.s. }}{\longrightarrow} \varphi^{B}(\omega) .
$$

Obviously $\varphi^{B}$ must be essentially bounded in $[0,1]$ and so integrable. Integrating (19) over any measurable set $A \in \mathcal{A}$ yields the result that, for $\bar{\lambda}^{\infty}$-a.e. $t^{\infty} \in T^{\infty}$, one has

$$
\frac{1}{n} \sum_{i=1}^{n} \int_{A} 1_{B}\left(g\left(t_{i}, \omega\right)\right) d P=\frac{1}{n} \sum_{i=1}^{n} P\left(A \cap g_{t_{i}}^{-1}(B)\right) \rightarrow \int_{A} \varphi^{B} d P .
$$

By Lemma 5.2, it follows that $t \mapsto P\left(A \cap g_{t}^{-1}(B)\right)$ is $\mathcal{T}$-measurable.

$\mathrm{T}(3) \Longrightarrow \mathrm{T}(1)$ : By Lemma 5.10, there is a $\mathcal{T} \otimes \mathcal{A}$-measurable mapping $(t, \omega) \mapsto \mu_{t \omega}$ from $T \times \Omega$ to $\mathcal{M}(X, \mathcal{B})$ such that for all $A \in \mathcal{A}$ and $B \in \mathcal{B}$, one has $P\left(A \cap g_{t}^{-1}(B)\right)=\int_{A} \mu_{t \omega}(B) d P$ for $\lambda$-a.e. $t \in T$. Next, part (1) of Lemma 5.8 implies that for any $B \in \mathcal{B}$, the mapping $(t, \omega) \mapsto \mu_{t \omega}(B)$ is $\mathcal{S}^{\mu} \otimes \mathcal{C}^{\mu}$-measurable, and thus $\mathcal{T} \otimes \mathcal{C}^{\mu}$-measurable. Lemma $5.11 \mathrm{implies}$ that $g$ admits an essentially regular conditional distribution process given the countably generated $\sigma$-algebra $\mathcal{C}^{\mu}$, and is essentially pairwise conditionally independent given $\mathcal{C}^{\mu}$.

$\mathrm{T}(1) \Longrightarrow \mathrm{P}(1)$ : If $\mathrm{T}(1)$ holds, we can apply Lemma 5.13 to each indicator function $1_{J}$ with $J \in \mathcal{T} \otimes \mathcal{B}$.

$\mathrm{T}(1) \Longrightarrow \mathrm{P}(4)$ : We apply Theorem 6.6 in $[19$, p. 47]. Because $X$ is a Polish space, so homeomorphic to a complete separable metric space, this theorem implies that there exist a topologically equivalent metric on $X$ and a sequence of bounded and uniformly continuous functions $\varphi_{m}: X \rightarrow \mathbb{R}$ $(m=1,2, \ldots)$ with the property that, for each $t^{\infty} \in T^{\infty}$ and each $\omega \in$ $\Omega$, the distribution $\nu_{t^{\infty}, \omega}^{n}$ converges weakly to $\tau_{\omega}$ if and only if the mean $\int_{X} \varphi_{m}(x) d \nu_{t^{\infty}, \omega}^{n}$ converges to $\int_{X} \varphi_{m}(x) d \tau_{\omega}$ as $n \rightarrow \infty$ for all $m=1,2, \ldots$ simultaneously.

For any Borel set $B$ in $X$, define $\tau_{\omega}(B)=\int_{T} \mu_{t \omega}(B) d \lambda$. For each fixed $m=1,2, \ldots$, because $\varphi_{m}$ is measurable and bounded, the definition of $\nu_{t^{\infty}, \omega}^{n}$ and Lemma 5.13 together imply that for $\bar{\lambda}^{\infty}$-a.e. sequence $t^{\infty} \in T^{\infty}$,

$$
\begin{aligned}
& \int_{X} \varphi_{m}(x) d \nu_{t^{\infty}, \omega}^{n}=\frac{1}{n} \sum_{i=1}^{n} \varphi_{m}\left(g\left(t_{i}, \omega\right)\right) \\
\underset{P-\text { a.s. }}{\longrightarrow} & \int_{T} \int_{X} \varphi_{m}(x) d \mu_{t \omega} d \lambda=\int_{X} \varphi_{m} d \tau_{\omega} .
\end{aligned}
$$


After excluding countably many $\bar{\lambda}^{\infty}$-null sets, there exists a subset $T_{1}^{\infty}$ of $T^{\infty}$ with $\bar{\lambda}^{\infty}\left(T_{1}^{\infty}\right)=1$ such that for each sequence $t^{\infty} \in T_{1}^{\infty}$, Equation (21) holds for all $m$ simultaneously. Consider any fixed sequence $t^{\infty} \in T_{1}^{\infty}$. Again, after excluding countably many $P$-null sets, for $P$-almost all $\omega \in \Omega$ one has $\int_{X} \varphi_{m}(x) d \nu_{t^{\infty}, \omega}^{n} \rightarrow \int_{X} \varphi_{m}(x) d \tau_{\omega}$ for all $m$ simultaneously. This implies that for each sequence $t^{\infty} \in T_{1}^{\infty}$, the sufficient condition for $\nu_{t^{\infty}, \omega}^{n}$ to converge weakly to $\tau_{\omega}$ is satisfied for $P$-almost all $\omega \in \Omega$.

$\mathrm{P}(4) \Longrightarrow \mathrm{T}(3)$ : Fix any $A \in \mathcal{A}$ with $P(A)>0$ and any bounded continuous function $\varphi$ on $X$. By $\mathrm{P}(4)$, for $\bar{\lambda}^{\infty}$-a.e. sequence $t^{\infty} \in T^{\infty}$ one has

$$
\int_{X} \varphi(x) d \nu_{t^{\infty}, \omega}^{n}=\frac{1}{n} \sum_{i=1}^{n} \varphi\left(g\left(t_{i}, \omega\right)\right) \underset{P-\text { a.s. }}{\longrightarrow} \int_{X} \varphi(x) d \tau_{\omega} .
$$

This obviously implies that

$$
\frac{1}{n} \sum_{i=1}^{n} \int_{A} \varphi\left(g\left(t_{i}, \omega\right)\right) d P \rightarrow \int_{A}\left[\int_{X} \varphi(x) d \tau_{\omega}\right] d P
$$

and so, by Lemma 5.2, that $t \mapsto \int_{A} \varphi\left(g_{t}(\omega)\right) d P$ is $\mathcal{T}$-measurable. Let $\mathcal{A}^{A}=$ $\{C \in \mathcal{A}: C \subseteq A\}$, let $P^{A}$ be the (conditional) probability measure defined on $\left(A, \mathcal{A}^{A}\right)$ by $P^{A}(E):=P(A \cap E) / P(A)$, and let $g^{A}$ denote the restriction of $g$ to $T \times A$. Then $\int_{A} \varphi\left(g_{t}(\omega)\right) d P=\int_{X} \varphi(x) d\left(P^{A}\left(g_{t}^{A}\right)^{-1}\right)$ for each $t$, so the mapping $t \mapsto \int_{X} \varphi(x) d P^{A}\left(g_{t}^{A}\right)^{-1}$ is $\mathcal{T}$-measurable. Note that a mapping $t \mapsto \psi_{t}$ from $T$ to $\mathcal{M}(X, \mathcal{B})$ is $\mathcal{T}$-measurable w.r.t. the Borel $\sigma$-algebra on $\mathcal{M}(X, \mathcal{B})$ generated by the topology of weak convergence of measures if and only if $\int_{X} \varphi d \psi_{t}$ is $\mathcal{T}$-measurable for every bounded continuous function $\varphi$ on $X$. This implies that $t \mapsto P^{A}\left(g_{t}^{A}\right)^{-1}$ defines a $\mathcal{T}$-measurable mapping from $T$ to $\mathcal{M}(X, \mathcal{B})$. By Lemma 1 in [9], therefore, given any fixed $B \in \mathcal{B}$, the mapping $t \mapsto P\left(A \cap g_{t}^{-1}(B)\right)=\lambda(A) \cdot P^{A}\left(g_{t}^{A}\right)^{-1}(B)$ is $\mathcal{T}$-measurable.

The above paragraphs have shown that $\mathrm{P}(1) \Longrightarrow \mathrm{P}(2), \mathrm{P}(2) \Longrightarrow \mathrm{P}(3)$, $\mathrm{P}(3) \Longrightarrow \mathrm{T}(3), \mathrm{T}(3) \Longrightarrow \mathrm{T}(1), \mathrm{T}(1) \Longrightarrow \mathrm{P}(1), \mathrm{T}(1) \Longrightarrow \mathrm{P}(4)$, and finally that $\mathrm{P}(4) \Longrightarrow \mathrm{T}(3)$. Given that $\mathrm{P}(1)$ and $\mathrm{T}(2)$ are identical, it follows that all the conditions in both Proposition 3.2 and Theorem 1 are equivalent. To finish the proof requires showing that when $g$ satisfies one (and thus all) of these conditions, then given any countably generated sub- $\sigma$-algebra $\mathcal{C}$ of $\mathcal{A}$, the process $g$ also has a stochastic macro structure given $\mathcal{C}$ if and only if the Monte Carlo $\sigma$-algebra satisfies $\mathcal{C}^{g} \subseteq \mathcal{C}$.

First, if $g$ has a stochastic macro structure given $\mathcal{C}$, then by Definition 3.3 there is an essentially regular conditional distribution process $\mu: T \times$ $\Omega \rightarrow \mathcal{M}(X, \mathcal{B})$ that is $\mathcal{T} \otimes \mathcal{C}$-measurable. Now apply Lemma 5.13 with 
$f(t, x)=1_{S}(t) \cdot 1_{B}(x)$ to show that the Monte Carlo limit function $\gamma(S, B, \omega)$ in Definition 3.1 is given by $\int_{S} \mu_{t \omega}(B) d \lambda$, which must be $\mathcal{C}$-measurable as a function of $\omega$. Since $\mathcal{C}^{g}$ is defined as the sub- $\sigma$-algebra of $\mathcal{A}$ generated by the family of functions $\omega \mapsto \gamma(S, B, \omega)(S \in \mathcal{T}, B \in \mathcal{B})$, we must have $\mathcal{C}^{g} \subseteq \mathcal{C}$.

Conversely, suppose that $\mathrm{T}(3)$ is true and that $\mathcal{C}$ contains $\mathcal{C}^{g}$. Following the proof that $\mathrm{T}(3) \Longrightarrow \mathrm{T}(1)$, there is a $\mathcal{T} \otimes \mathcal{A}$-measurable mapping $(t, \omega) \mapsto$ $\mu_{t \omega}$ from $T \times \Omega$ to $\mathcal{M}(X, \mathcal{B})$ such that, for all $A \in \mathcal{A}$ and $B \in \mathcal{B}$, one has $P\left(A \cap g_{t}^{-1}(B)\right)=\int_{A} \mu_{t \omega}(B) d P$ for $\lambda$-a.e. $t \in T$; moreover, that mapping must be $\mathcal{T} \otimes \mathcal{C}^{\mu}$-measurable. Then Lemma 5.11 implies that $g$ has a stochastic macro structure given $\mathcal{C}^{\mu}$. Lemma 5.13 and the argument in the above paragraph imply that $\gamma(S, B, \omega)=\int_{S} \mu_{t \omega}(B) d \lambda$ for $S \in \mathcal{T}$ and $B \in \mathcal{B}$. It follows from part (2) of Lemma 5.8 and from Definition 5.7 that $\mathcal{C}^{\mu} \subseteq \mathcal{C}^{g}$, so $\mathcal{C}^{\mu} \subseteq \mathcal{C}$ by hypothesis. Hence, $\mu$ is also $\mathcal{T} \otimes \mathcal{C}$-measurable. Then Lemma 5.11 implies that $g$ has a stochastic macro structure given $\mathcal{C}$. Also, since $g$ has a stochastic macro structure given $\mathcal{C}^{\mu}$, the previous paragraph shows that $\mathcal{C}^{\mu}$ contains $\mathcal{C}^{g}$, so $\mathcal{C}^{\mu}=\mathcal{C}^{g}$. By Lemma 5.9, for each $J \in \mathcal{T} \otimes \mathcal{B}$ the mapping $\omega \mapsto \gamma_{\omega}(J)$ is $\mathcal{C}^{g}$-measurable. Hence, Lemma 5.13 implies that $\mathcal{C}^{g}$ is also the smallest sub- $\sigma$-algebra of $\mathcal{A}$ with respect to which the mapping $\omega \mapsto \gamma_{\omega}(J)$ is measurable for every $J \in \mathcal{T} \otimes \mathcal{B}$.

\subsection{Proof of Proposition 3.4}

For each $(t, \omega) \in T \times \Omega$, let $\mu_{t \omega}^{\prime}$ be the Dirac measure $\delta_{g(t, \omega)}$ at $g(t, \omega)$. Because of the hypothesis that $g$ is $\mathcal{T} \otimes \mathcal{A}$-measurable, so is $\mu^{\prime}$. For any $A \in \mathcal{A}$ and $B \in \mathcal{B}$, it is clear that $\mu_{t \omega}^{\prime}(B)=1_{g_{t}^{-1}(B)}(\omega)$, so

$$
P\left(A \cap g_{t}^{-1}(B)\right)=\int_{A} \mu_{t \omega}^{\prime}(B) d P \text { for } \lambda \text {-almost all } t \in T .
$$

By Theorem 1, the process $g$ has event-wise measurable conditional probabilities. Thus the uniqueness property in Lemma 5.10 implies that $\mu_{t \omega}^{\prime}=\mu_{t \omega}$ for $(\lambda \times P)$-almost all $(t, \omega) \in T \times \Omega$. Fix any $B \in \mathcal{B}$. The last part of the proof of Theorem 1 implies that $\mu_{t \omega}(B)$ (and so $\mu_{t \omega}^{\prime}(B)$ ) is $\mathcal{T} \otimes \mathcal{C}^{g}$ measurable as a function of $(t, \omega)$. So the set $g^{-1}(B)$ is $\mathcal{T} \otimes \mathcal{C}^{g}$-measurable. This is true for any $B \in \mathcal{B}$, so $g$ is $\mathcal{T} \otimes \mathcal{C}^{g}$-measurable. The Fubini property then implies that $g_{t}$ is $\mathcal{C}^{g}$-measurable for $\lambda$-almost all $t \in T$. 


\subsection{Proof of the results in Section 4}

\section{Proof of Proposition 4.1:}

$(1) \Longrightarrow(3)$ : The condition of Lemma 5.13 is obviously satisfied with $\mathcal{C}=$ $\{\Omega, \emptyset\}$ and $\mu_{t \omega}=\mu_{t}$. So the Monte Carlo limit measure $\gamma$ on $(T \times X, \mathcal{T} \otimes \mathcal{B})$ is defined by $\gamma(J):=\int_{T} \mu_{t}\left(J_{t}\right) d \lambda$ for each $J \in \mathcal{T} \otimes \mathcal{B}$.

$(3) \Longrightarrow(2)$ : This is obvious. ${ }^{13}$

$(2) \Longrightarrow(1)$ : By Proposition 3.2 and Theorem 1, the mapping $t \mapsto P(A \cap$ $\left.g_{t}^{-1}(B)\right)$ is $\mathcal{T}$-measurable. For each $S \in \mathcal{T}$ and $B \in \mathcal{B}$, and for $\bar{\lambda}^{\infty}$-a.e. sequence $t^{\infty} \in T^{\infty}$, one has

$$
\frac{1}{n} \sum_{i=1}^{n} 1_{S}\left(t_{i}\right) 1_{B}\left(g\left(t_{i}, \omega\right)\right) \underset{P-\text { a.s. }}{\longrightarrow} \int_{S} \mu_{t}(B) d \lambda .
$$

Integrating (5) over any measurable set $A \in \mathcal{A}$ yields the result that, for $\bar{\lambda}^{\infty}$-a.e. $t^{\infty} \in T^{\infty}$, one has

$$
\begin{aligned}
\frac{1}{n} \sum_{i=1}^{n} 1_{S}\left(t_{i}\right) \int_{A} 1_{B}\left(g\left(t_{i}, \omega\right)\right) d P & =\frac{1}{n} \sum_{i=1}^{n} 1_{S}\left(t_{i}\right) P\left(A \cap g_{t_{i}}^{-1}(B)\right) \\
& \rightarrow P(A) \int_{S} \mu_{t}(B) d \lambda .
\end{aligned}
$$

By the classical law of large numbers, $\frac{1}{n} \sum_{i=1}^{n} 1_{S}\left(t_{i}\right) P\left(A \cap g_{t_{i}}^{-1}(B)\right) \rightarrow$ $\int_{S} P\left(A \cap g_{t}^{-1}(B)\right) d \lambda$ for $\lambda^{\infty}$-a.e. $t^{\infty} \in T^{\infty}$. For any $S \in \mathcal{T}, A \in \mathcal{A}$ and $B \in \mathcal{B}$, therefore,

$$
\int_{S} P\left(A \cap g_{t}^{-1}(B)\right) d \lambda=P(A) \int_{S} \mu_{t}(B) d \lambda=\int_{S} \int_{A} \mu_{t}(B) d P d \lambda .
$$

By the essential uniqueness of the Radon-Nikodym derivative, Equation (25) implies that $P\left(A \cap g_{t}^{-1}(B)\right)=\int_{A} \mu_{t}(B) d P$ for $\lambda$-a.e. $t \in T$. So by Lemma $5.10, \mu_{t \omega}=\mu_{t}$, which is $\mathcal{T} \otimes\{\Omega, \emptyset\}$-measurable. Part (1) then follows from Lemma 5.11.

\section{Proof of Proposition 4.2:}

$(1) \Longrightarrow(2)$ : This follows from Lemma 5.11.

$(2) \Longrightarrow(4)$ : This follows from Lemma 5.13.

$(4) \Longrightarrow(3)$ : This follows directly from the definitions.

$(3) \Longrightarrow(1)$ : For any $S \in \mathcal{T}, A \in \mathcal{A}$ and $B \in \mathcal{B}$, we repeat the procedure used to prove $(2) \Longrightarrow(1)$ in Proposition 4.1 . The only difference

\footnotetext{
${ }^{13}$ Note that $(1) \Longrightarrow(2)$ was already shown after the statement of Proposition 4.1. Here we prove $(1) \Longrightarrow(3)$ instead, while $(3) \Longrightarrow(2)$ is trivial.
} 
is that the limit in Equation (24) becomes $\lambda(S) \int_{A} \mu_{\omega}(B) d P$ instead of $P(A) \int_{S} \mu_{t}(B) d \lambda$. Thus, we obtain

$$
\int_{S} P\left(A \cap g_{t}^{-1}(B)\right) d \lambda=\int_{S} \int_{A} \mu_{\omega}(B) d P d \lambda .
$$

Essential uniqueness of the Radon-Nikodym derivative then implies (1).

\section{Proof of Proposition 4.4:}

$(1) \Longrightarrow(2)$ : This follows from Lemma 5 in [9]. Note that equations (5) and (6) in the proof of Lemma 5 in [9] follow from essential pairwise exchangeability (as defined in part (1) of Definition 4.3 of this paper) and from the Fubini property for the measure $\bar{\lambda}^{2}$.

$(2) \Longrightarrow(3)$ : This is a special case of Lemma 5.10, which is also shown in Lemma 4 of [9] under the special assumption of pairwise measurable probabilities.

$(3) \Longrightarrow(4)$ : This follows trivially from Proposition 4.2.

$(4) \Longrightarrow(1)$ : The proof of Lemma 7 in [9] can be repeated, but with $\lambda \times \lambda$ replaced by $\bar{\lambda}^{2}$.

\section{References}

[1] P. Billingsley, Probability and Measure, (3rd. edn.), John Wiley, New York, 1995.

[2] W. W. Bledsoe and A. P. Morse, Product measures, Transactions of the American Mathematical Society 79 (1955), 173-215.

[3] Y. S. Chow and H. Teicher, Probability Theory: Independence, Interchangeability, Martingales, (3rd. edn.), Springer, New York, 1997.

[4] G. M. Constantinides and D. Duffie Asset pricing with heterogeneous consumers, Journal of Political Economy 104 (1996), 219-40.

[5] J. L. Doob, Stochastic processes depending on a continuous parameter, Transactions of the American Mathematical Society 42 (1937), 107-140.

[6] J. L. Doob, Stochastic Processes, John Wiley, New York, 1953.

[7] R. M. Dudley, Real Analysis and Probability, Chapman \& Hall, New York, 1989.

[8] R. Durrett, Probability: Theory and Examples, (2nd. edn.), Wadsworth, Belmont, California, 1996. 
[9] P. J. Hammond and Y. N. Sun, Monte Carlo simulation of macroeconomic risk with a continuum of agents: The symmetric case, Economic Theory 21 (2003), 743-766.

[10] P. J. Hammond and Y. N. Sun, Joint measurability and the one-way Fubini property for a continuum of independent random variables, Proceedings of the American Mathematical Society 134 (2006), 737-747.

[11] P. J. Hammond and Y. N. Sun, The essential equivalence of pairwise and mutual conditional independence, Probability Theory and Related Fields 135 (2006), 415-427.

[12] J. Heaton and D. J. Lucas, Evaluating the effects of incomplete markets on risk sharing and asset pricing, Journal of Political Economy 104 (1996), 443-487.

[13] J. Hoffmann-Jørgensen, The law of large numbers for non-measurable and non-separable random elements, Astérisque 131 (1985), 299-356.

[14] M. O. Jackson and I. Kremer, Envy-freeness and implementation in large economies, Review of Economic Design, forthcoming.

[15] K. Judd, The law of large numbers with a continuum of IID random variables. Journal of Economic Theory 35 (1985), 19-25.

[16] O. Kallenberg, Foundations of Modern Probability, (2nd. edn.), Springer, New York, 2002.

[17] R. McLean and A. Postlewaite, Informational size and incentive compatibility, Econometrica 70 (2002), 2421-2453.

[18] T. Palfrey and S. Srivastava, Private information in large economies, Journal of Economic Theory 39 (1986), 34-58.

[19] K. R. Parthasarathy, Probability Measures on Metric Spaces, New York, Academic Press, 1967.

[20] Y. N. Sun, A theory of hyperfinite processes: The complete removal of individual uncertainty via exact LLN, Journal of Mathematical Economics 29 (1998), 419-503.

[21] Y. N. Sun, The almost equivalence of pairwise and mutual independence and the duality with exchangeability, Probability Theory and Related Fields 112 (1998), 425-456. 
[22] Y. N. Sun, The exact law of large numbers via Fubini extension and characterization of insurable risks, Journal of Economic Theory 126 (2006), 31-69.

[23] Y. N. Sun and N. C. Yannelis, Perfect competition in asymmetric information economies: Compatibility of efficiency and incentives, Journal of Economic Theory 134 (2007), 175-194. 